\title{
MATHEMATICAL ANALYSIS OF A DIFFUSIVE PREDATOR-PREY MODEL WITH HERD BEHAVIOR AND PREY ESCAPING
}

\author{
Fethi Souna $^{1}$, SAlih DJILAli ${ }^{2,3, *}$ And FAyssal Charif ${ }^{4,5}$
}

\begin{abstract}
In this paper, we consider a new approach of prey escaping from herd in a predator-prey model with the presence of spatial diffusion. First, the sensitivity of the equilibrium state density with respect to the escaping rate has been studied. Then, the analysis of the non diffusive system was investigated where boundedness, local, global stability, Hopf bifurcation are obtained. Besides, for the diffusive system, we proved the occurrence of Hopf bifurcation and the non existence of diffusion driven instability. Furthermore, the direction of Hopf bifurcation has been proved using the normal form on the center manifold. Some numerical simulations have been used to illustrate the obtained results.
\end{abstract}

Mathematics Subject Classification. 92B05, 35K57, 34D20.

Received December 27, 2018. Accepted October 2, 2019.

\section{INTRODUCTION}

It is well known that the predator-prey model became a subject of interest of research activities in ecological systems. For the past century a huge number of models introduced to the wide audience for the subject of modeling a specific manner of interaction between prey and predator see for instance the papers [7, 20, 21]. The crucial component of the relationship between the prey population and the predator population in real life interaction is the functional response, which it changes from one population to another, it is the reason for having many interaction functionals such as Holling I-III functional response [13, 15], Holling-Tanner functional response [23], Beddington-Deangelis functional response [14], Ratio-dependent functional response [16], so on.

However, the mentioned classical functional responses have the certain limitations to model many realistic behavior in ecology. For discussing some of this limitations we introduce the following prototypical predator-prey model (Lotka-Volterra model [2])

$$
\left\{\begin{array}{l}
\frac{\mathrm{d} u}{\mathrm{~d} t}=r u\left(1-\frac{u}{k}\right)-a u v \\
\frac{\mathrm{d} v}{\mathrm{~d} t}=-m v+e a u v
\end{array}\right.
$$

Keywords and phrases: Herd behavior, predator-prey model, Hopf bifurcation, prey escaping, spatial diffusion.

1 Biomathematics Laboratory, University of Djillali Liabes, Sidi-Bel-Abbès, Algeria.

2 Faculty of Exact Sciences and Informatics, Mathematic Department, University of Hassiba Benbouali, Chlef, Algeria.

${ }^{3}$ Laboratoire d'Analyse Non Linéaire et Mathématiques Appliquées, Université de Tlemcen, Tlemcen, Algeria.

${ }^{4}$ Faculty of sciences, Mathematic Department, University of Moulay Tahar, Saida, Algeria.

${ }^{5}$ Laboratoire de Géométrie, Analyse, Contrôle et Applications, University of Moulay Tahar, Saida, Algeria.

* Corresponding author: djilali.salih@yahoo.fr 
where $u(t)$ and $v(t)$ represents respectively the sizes of prey and the predator populations at the time $t, r$ is the intrinsic growth rate, $k$ describes the carrying capacity of the environment for the prey population, $0<e<1$ is the conversion rate of prey biomass into predator biomass, $m$ is the natural death rate for the predator population which has been assumed to be constant, $a$ represents the maximum value at which the reduction rate per capita of the prey population can be attained. It is well known that $h(u, v)=a u v$ is the Holling I functional response which models the quality of the interaction between the prey population and the predator population. Besides, $h(u, 1)=a u$ is the number of the consumed prey by one predator, which shows that the prey population has a solitary behavior.

On the other hand, the prey population behavior in the presence of social behavior has been recently modeled, which can be seen in the real life when they all gather in a huge herd. This point of view has been put due to the papers $[1,28]$, where the investigated model is as follows

$$
\left\{\begin{array}{l}
\frac{\mathrm{d} u}{\mathrm{~d} t}=r u\left(1-\frac{u}{k}\right)-a \sqrt{u} v \\
\frac{\mathrm{d} v}{\mathrm{~d} t}=-m v+e a \sqrt{u} v \\
u(0)=u_{0} \geq 0, v(0)=v_{0} \geq 0
\end{array}\right.
$$

where the functional response $h(u, v)=a \sqrt{u} v$ represents the interaction between the prey population and the predator population in the presence of herd behavior. For better explanation of this functional response we take a simple example when the prey population has a social behavior with a regular shape such as circle or a square, obviously the predator hunt only on the outer bound of the prey pack. In this case, the number of the captured prey by one predator will be proportional to the number of the prey population on the boundary of the prey herd, which means that is proportional to the square root of the prey population density; it is the main reason of proposing such as functional response. This functional response has been used widely, we cite for instance the papers $[6,27,29,30,32,35,36]$. It follows by a several of functional responses which models this interaction by including many factors such as herd shape, predator average handling time of the prey on the bounders, we take as example the papers $[3,7,9,10,19,24,26,33,38]$.

In the real world, during the predator hunting of the prey, it is almost impossible for the prey population to keep the pack all together, due to the panic of some of them. The attack of the predator will push the prey pack to split into two groups. The first will stay in the group, and the second will leave the pack and go in any direction, this second has a solitary behavior and it is the most preferred prey to the predator. In the present paper, we will consider that there is a constant proportional density of the prey population denoted by $0<P<1$ which will abandon the prey pack during the hunting for the reason of panic, which can be called also by escaping rate, and the other $1-P$ stays in the pack. For modeling this behavior, there are two ways for the predator to consume the prey. The first, is to hunt on the boundary of the prey pack which means $\sqrt{(1-P) u}$ (is the density of the prey on the outer bound of the pack) or hunt the escaping prey which means $P u$. According to the above discussion, we propose the following model

$$
\left\{\begin{array}{l}
\frac{\mathrm{d} u}{\mathrm{~d} t}=r u\left(1-\frac{u}{k}\right)-a_{1} \sqrt{(1-P) u} v-a_{2} P u v \\
\frac{\mathrm{d} y}{\mathrm{~d} t}=-m v+e a_{1} \sqrt{(1-P) u} v+e a_{2} P u v, \\
u(0)=u_{0} \geq 0, v(0)=v_{0} \geq 0,
\end{array}\right.
$$

where $a_{1}, a_{2}$ represents the maximum value at which per capita reduction rate of the prey population in pack and solitary prey can attained, respectively. For $a_{1}=a_{2}$ represents the non selective hunting of the prey by a predator, and for $a_{1} \neq a_{2}$ shows the predator preference of one prey on another (if $a_{1}>a_{2}$ shows the predator preference of the solitary prey on the prey in the pack). 
Remark 1.1. It is easy to see that for $P=0$ (there is no escaping), the system (1.3) becomes the system (1.2). Besides, if $P=1$ (all the prey escape, which means that there is no herd behavior) then the system (1.3) becomes the system (1.1). Furthermore, we proved the existence of a new functional response which describes the interaction predator-prey in the presence of herd behavior and prey escaping from the herd. The proposed functional response is $h(u, v)=a_{1} \sqrt{(1-P) u} v+a_{2} P u v$.

In the real world, the prey and the predator are always in movement, which can be modeled by the presence of self-disperse. The spatial diffusion has been widely studied in literature, see [17, 24-27, 32, 34-37]. In the considered paper, the study of the system (1.3) and the effect of the spatial diffusion on the system (1.3) has been investigated. If we assume that the two considered populations are always in movement (in $\mathbb{R}^{2}$ or $\mathbb{R}^{3}$ ) thus each one of those population will follow a trajectory (path). The length of this path we denoted by $x$. This point of view is a strong simplification of the general case, where it has been used widely we cit for instance the papers [7, 25-27]. Besides, we will assume that the two populations are in isolated patches, which can be elaborated using the zero flux boundary condition. The system (1.3) becomes in the presence of spatial diffusion:

$$
\left\{\begin{array}{l}
u_{t}-\mathrm{d} u_{x x}=r u\left(1-\frac{u}{k}\right)-a_{1} \sqrt{(1-P) u} v-a_{2} P u v \\
v_{t}-\tilde{\mathrm{d}} v_{x x}=-m v+e a_{1} \sqrt{(1-P) u} v+e a_{2} P u v, \\
u_{x}(0, t)=u_{x}(l \pi, t)=v_{x}(0, t)=v_{x}(l \pi, t)=0 \quad \forall t \geq 0 \\
u(x, 0)=\phi(x) \geq 0 \quad v(x, 0)=\psi(x) \geq 0 \quad x \in(0, l \pi)
\end{array}\right.
$$

where $x$ is the location of the prey or the predator at the time $t$, and $l \pi$ is the domain size, $d, \tilde{d}$ are the diffusion rates for the prey and the predator, respectively. The homogeneous Neumann boundary conditions represents that the prey and the predator move with a distance between 0 and $l \pi$. For more examples see $[8,11,18,22]$.

It is easy to check that the homogeneous steady states of the system $(1.4)$ are $(0,0),(k, 0)$ and $\left(u^{*}, v^{*}\right)$ where

$$
\left\{\begin{array}{l}
u^{*}=\left(\frac{e a_{1} \sqrt{(1-P)}+\sqrt{\left(e a_{1} \sqrt{(1-P)}\right)^{2}+4 m e a_{2} P}}{2 e a_{2} P}\right)^{2}, \quad \text { exists if and only if } k>u^{*} . \\
v^{*}=\frac{r \sqrt{u^{*}}}{a_{1} \sqrt{(1-P)}+a_{2} P \sqrt{u^{*}}}\left(1-\frac{u^{*}}{k}\right)>0 .
\end{array}\right.
$$

The rest of the paper is organized as follows. In Section 2, we focus on studying the effect of the escaping rate on the positive equilibrium state $\left(u^{*}, v^{*}\right)$. In the next section the existence of a priori bound of the solution, the global stability of the semi trivial equilibrium $(k, 0)$, and the occurrence of Hopf bifurcation for the system (1.3) have been proved. In Section 4 the analysis of the diffusive system has been successfully studied where the local stability and the occurrence of Hopf bifurcation have been shown. Furthermore, the stability of the homogeneous and non homogeneous periodic solutions have been established using the normal form on the center of manifold. An extend numerical simulations have been carried out to insure the theoretical results. A discussion section ends the paper.

\section{Sensitivity ANALYSis}

In this section, we will study the impact of the prey escaping on the equilibrium densities of both the prey and the predator populations. First, we differentiate the density equilibrium of the prey population with respect to $P$ we obtain

$$
\frac{\mathrm{d} u^{*}}{\mathrm{~d} P}=\left(\frac{e a_{1} \sqrt{(1-P)}+\sqrt{\left(e a_{1} \sqrt{(1-P)}\right)^{2}+4 m e a_{2} P}}{2 e a_{2} P}\right)\left(\frac{a_{1}(P-2)}{a_{2} P^{2} \sqrt{1-P}}-\frac{4 m a_{2} P+e a_{1}^{2}(1-P)+e a_{1}^{2}}{\sqrt{e} a_{2} P^{2} \sqrt{e a_{1}^{2}+P\left(4 m a_{2}-e a_{1}^{2}\right)}}\right)<0 .
$$




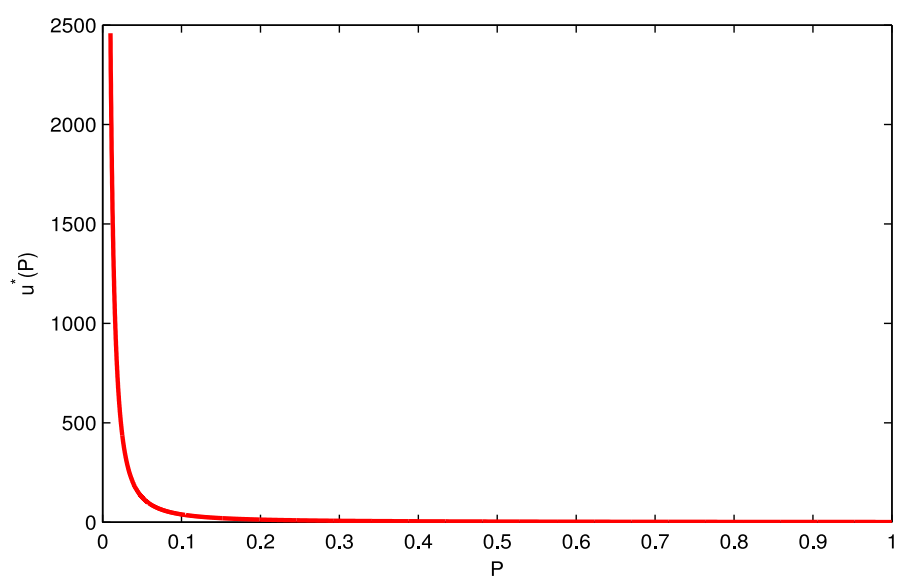

FIGURE 1. The negative effect of the prey escaping on the prey density equilibrium state for the values $a_{1}=1 ; a_{2}=2.1 ; e=0.44 ; m=1.01 ; r=0.2, k=8000$.

Obviously, the prey equilibrium density is decreasing with respect to the escaping rate, means that the prey escaping has a negative effect on the prey equilibrium density. Which shows the importance of the social behavior for the prey population. Figure 1 shows the effect of the variable $P$ on the prey density equilibrium state.

Now focusing on studying the impact of the escaping rate $P$ on the predator density equilibrium state. The predator density equilibrium can be written as follows

$$
v^{*}=\frac{e r u^{*}}{m}\left(1-\frac{u^{*}}{k}\right)
$$

By a differentiation of the predator density equilibrium state (2.2) with respect to the escaping rate $P$ we obtain

$$
\begin{aligned}
\frac{\mathrm{d} v^{*}}{\mathrm{~d} P} & =\frac{e r}{m} \frac{\mathrm{d} u^{*}}{\mathrm{~d} P}\left(1-\frac{2 u^{*}}{k}\right), \\
& =\frac{2 e r}{k m} \frac{\mathrm{d} u^{*}}{\mathrm{~d} P}\left(\frac{k}{2}-u^{*}\right) .
\end{aligned}
$$

In order to study the positivity of $\frac{k}{2}-u^{*}$ we draw the following lemma

Lemma 2.1. (i) If $k \leq \frac{2 m}{e a_{2}}$ then $\frac{k}{2}-u^{*}<0$ for any $0<P<1$.

(ii) if $k>\frac{2 m}{e a_{2}}$ then there exists $0<P_{c r}<1$ such that $\frac{k}{2}-u^{*}<0$ for $0<P<P_{c r}$ and $\frac{k}{2}-u^{*}>0$ for $P_{c r}<P<1$.

Proof. (i) It is not difficult to verify that $\lim _{P \rightarrow 0^{+}} u^{*}(P)=+\infty$ and $\lim _{P \rightarrow 1} u^{*}(P)=\frac{m}{e a_{2}}$ and using the fact that $\frac{\mathrm{d} u^{*}}{\mathrm{~d} P}<0$ then for $\frac{k}{2} \leq \frac{m}{e a_{2}}=\min _{p \in] 0,1]} u^{*}(P)$ we deduce that $\frac{k}{2}-u^{*}<0$ which completes the first part of the proof.

(ii) for $\frac{k}{2}>\frac{m}{e a_{2}}=\min _{p \in] 0,1]} u^{*}(P)$ then the curve of the functional $u^{*}(P), 0<P<1$ intersect the line $\frac{k}{2}$ at $0<P_{c r}<1$ then we have $\frac{k}{2}-u^{*}<0$ for $0<P<P_{c r}$ and $\frac{k}{2}-u^{*}>0$ for $P_{c r}<P<1$. The proof is completed. 

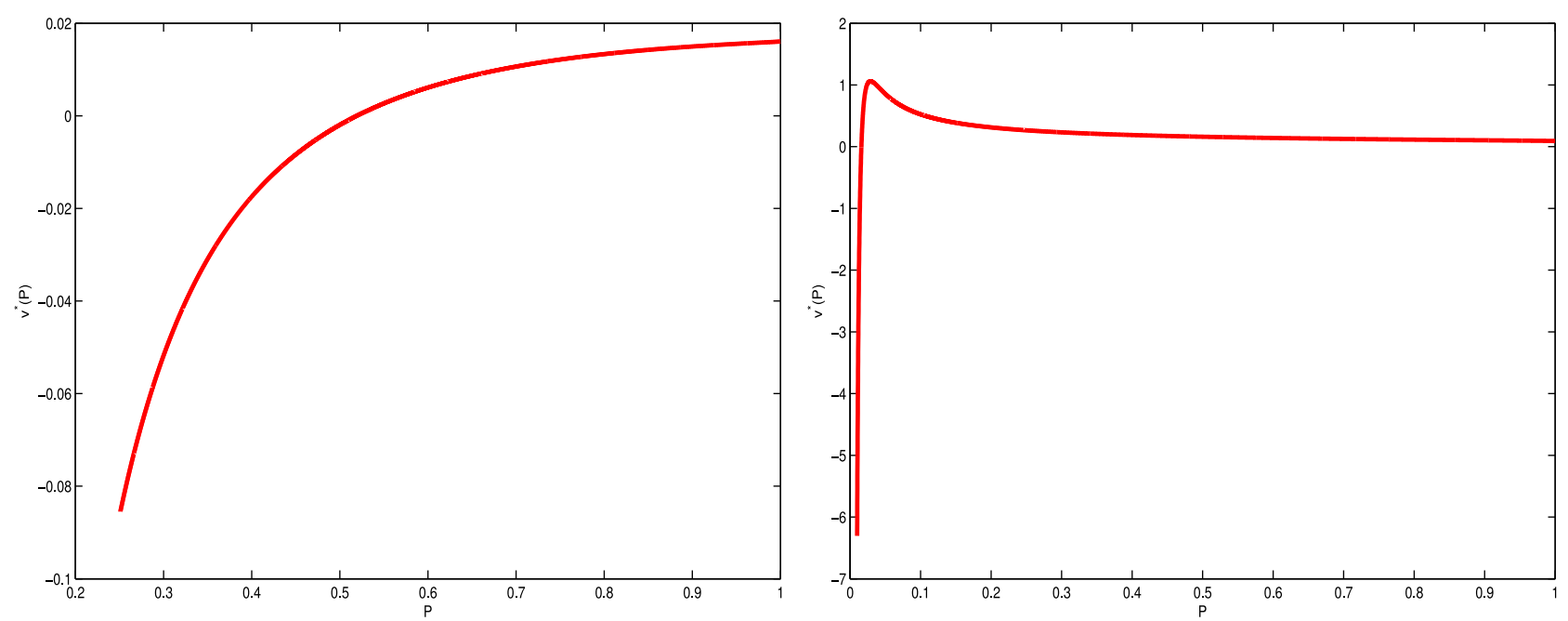

FiguRE 2. The effect of the prey escaping on the predator density equilibrium state for the values (i) (Left hand figure) the values $a_{1}=1.9 ; a_{2}=0.1 ; e=0.0044 ; m=2.01 ; r=0.2$, $k=10000<\frac{2 m}{e a_{2}}=91364$. (ii) (Right hand figure) the values $a_{1}=1 ; a_{2}=2.1 ; e=0.44$; $m=1.01 ; r=0.2, k=8000>\frac{2 m}{e a_{2}}=2.1861$.
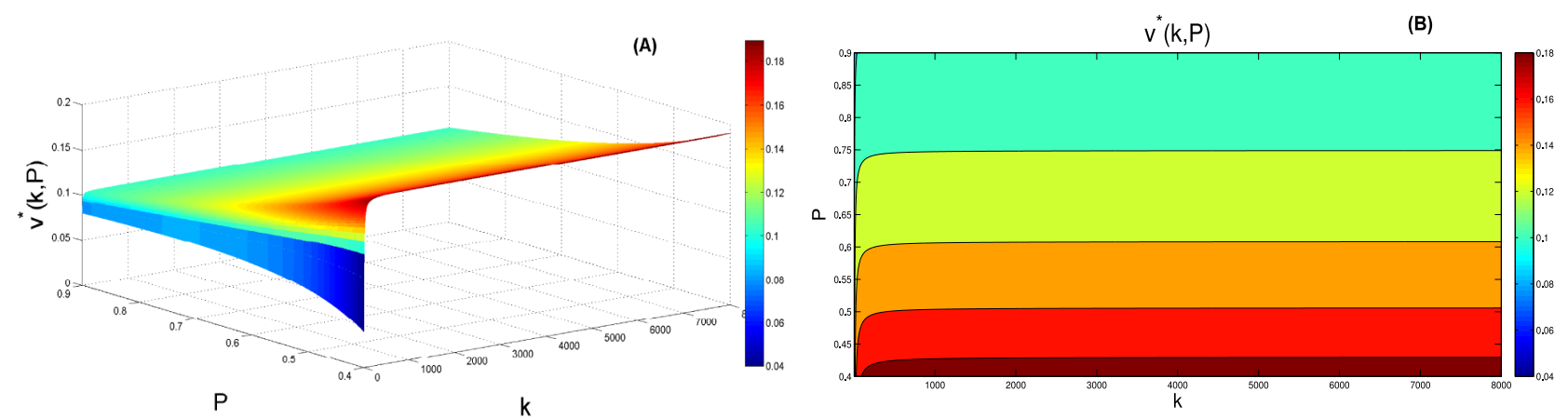

FIGURE 3. the impact of both the prey escaping and the carrying capacity of the space for the values $a_{1}=1 ; a_{2}=2.1 ; e=0.44 ; m=1.01 ; r=0.2$.

Using Lemma 2.1 together with the fact that $\frac{\mathrm{d} u^{*}}{\mathrm{~d} P}<0$, we draw the following results

(i) If $k \leq \frac{2 m}{e a_{2}}$ then $\frac{\mathrm{d} v^{*}}{\mathrm{~d} P}>0$ and based on the ecological meaning we deduce that the prey escaping has a positive impact on the predator density equilibrium.

(ii) If $k>\frac{2 m}{e a_{2}}$ then there exists $0<P_{c r}<1$ such that

$$
\frac{\mathrm{d} v^{*}}{\mathrm{~d} P}= \begin{cases}>0 & \text { for } 0<P<P_{c r} \\ <0 & \text { for } P_{c r}<P<1\end{cases}
$$




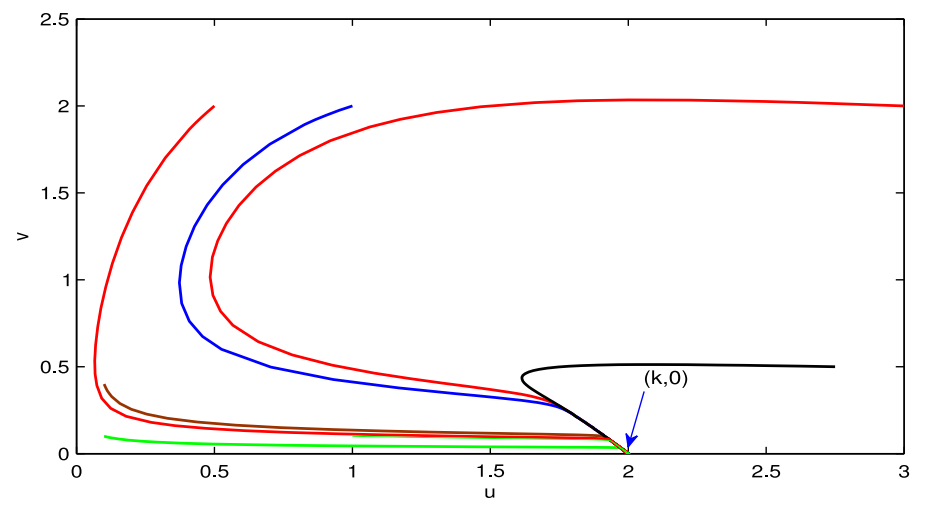

Figure 4. Phase portraits of the system $(1.3)$ when $\left(u^{*}, v^{*}\right)$ does not exists and $(k, 0)$ is globally asymptotically stable for $k=2<u^{*}=3.5871$.

which means that the prey escaping has a positive impact on the predator density equilibrium for $P<P_{c r}$ and a negative impact for $P_{c r}<P<1$ (see Fig. 2).

\section{StABILITY, BIFURCATION ANALYSIS OF THE NON SPATIAL SYSTEM}

This section is devoted to study the solution behavior for the model (1.3) where a priori bound of solution, global stability of the boundary equilibrium $(k, 0)$ has been investigated. Further, the existence of Hopf bifurcation has been shown.

In order to show the existence of the bounds of the system (1.3) we set the following theorem

Theorem 3.1. Let $(u(t), v(t))$ be the solution of the system (1.3) then

$$
\begin{aligned}
& \limsup _{t \rightarrow+\infty} u(t) \leq k, \\
& \limsup _{t \rightarrow+\infty} v(t) \leq \frac{m+r}{m} e k .
\end{aligned}
$$

Proof. Obviously, for the system (1.3) the positive invariant set is the first quadrant $\mathbb{R}_{+}^{2}$, since $u=0, v=0$ are its solutions. From the first equation of the system (1.3) we have

$$
u^{\prime}(t) \leq r u(t)\left(1-\frac{u(t)}{k}\right)
$$

Let $\tilde{u}(t)$ be the solution of the following initial value problem

$$
\left\{\begin{array}{l}
\frac{\mathrm{d} \tilde{u}(t)}{\mathrm{d} t}=r \tilde{u}(t)\left(1-\frac{u(t)}{k}\right), \\
\tilde{u}(0)=u(0),
\end{array}\right.
$$

using the standard comparison principle, we have $u(t) \leq \tilde{u}(t)$ for all $t \in[0,+\infty)$. Thus

$$
\limsup _{t \rightarrow+\infty} u(t) \leq \limsup _{t \rightarrow+\infty} \tilde{u}(t)=k .
$$

Now we put

$$
w(t)=e u(t)+v(t) .
$$



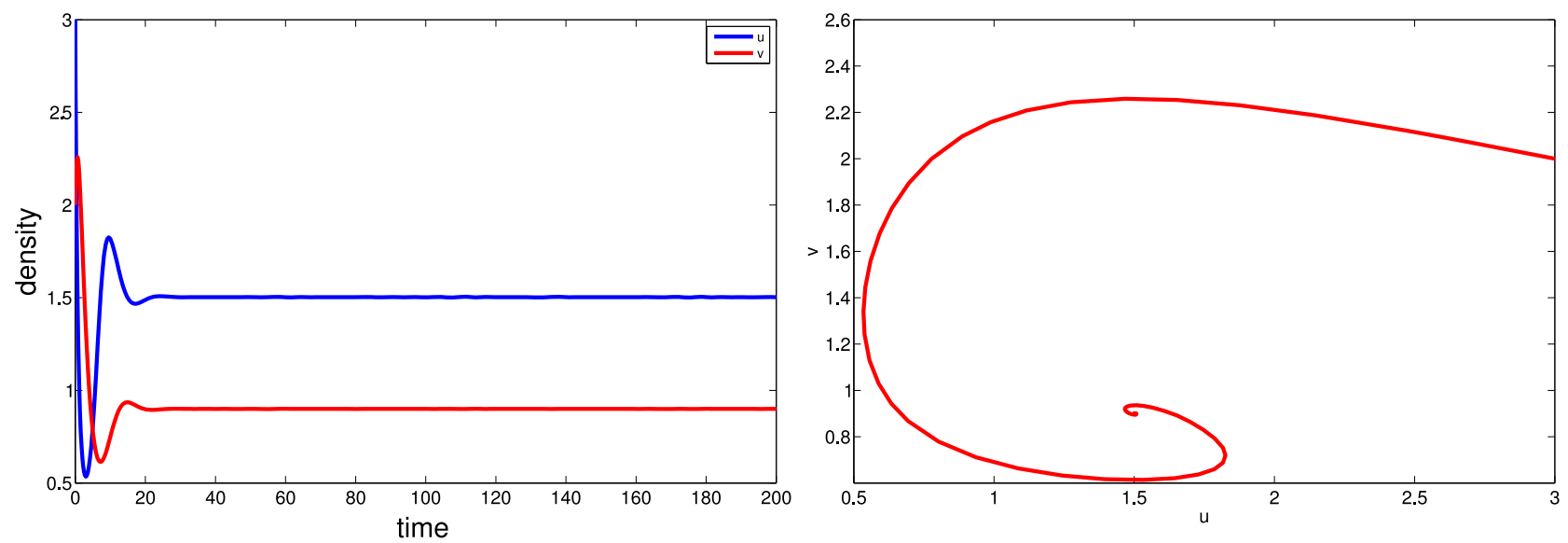

Figure 5. Trajectory and phase portraits of the system $(1.3)$ when $\left(u^{*}, v^{*}\right)=(1.55,0.89)$ is locally asymptotically stable and $k=2<k^{*}=17.0855$ for the values $P=0.5 ; a_{1}=0.2$; $a_{2}=1.1 ; e=0.5 ; m=0.5 ; r=1.2$.

then

$$
\begin{aligned}
\dot{w}(t) & =e \dot{u}(t)+\dot{v}(t), \\
& =-m v(t)+r u(t)\left(1-\frac{u(t)}{k}\right), \\
& =-m(e u(t)+v(t))+e m u(t)+r u(t)\left(1-\frac{u(t)}{k}\right),
\end{aligned}
$$

leads to

$$
\frac{\mathrm{d} w(t)}{\mathrm{d} t} \leq-m w(t)+e u(t)(m+r) .
$$

From $\limsup _{t \rightarrow+\infty} u(t) \leq k$ we can deduce that there exists $T>0$ such that for $t>T, u(t)<k$. Then for $t>T$ we have

$$
\frac{\mathrm{d} w(t)}{\mathrm{d} t} \leq-m w(t)+e k(m+r),
$$

by a similar argument we have

$$
\limsup _{t \rightarrow+\infty} v(t) \leq \frac{m+r}{m} e k
$$

which completes the proof.

Now, focusing on proving the global stability of the semi trivial equilibrium state $(k, 0)$. The following theorem summarize the obtained results

Theorem 3.2. Assume that $k<u^{*}$ then the semi trivial equilibrium is globally asymptotically stable.

Proof. The eigenvalues of Jacobian matrix of the system (1.3) on $(k, 0)$ are $\lambda_{1}=-r<0, \lambda_{2}=-m+e b_{1} \sqrt{k}+$ $e b_{2} k<0$ (for $k<u^{*}$ ) which means that $(k, 0)$ is locally asymptotically stable, it remain to prove that $(k, 0)$ is 

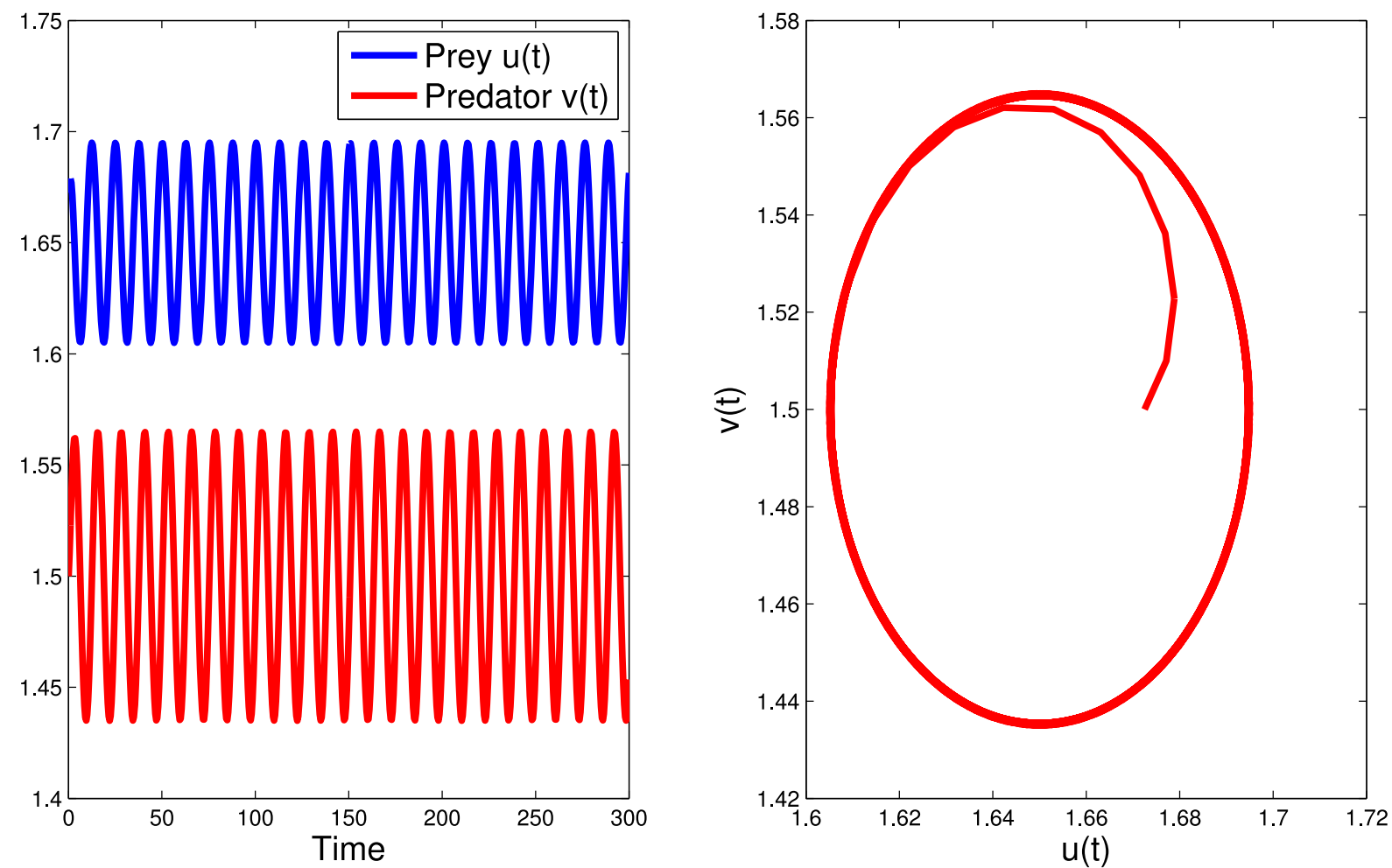

FIgURE 6. Trajectory and phase portraits of the system $(1.3)$ when $\left(u^{*}, v^{*}\right)=(1.51,1.65)$ is unstable and $k=18>k^{*}=17.0855$ for the values $P=0.5 ; a_{1}=0.2 ; a_{2}=1.1 ; e=0.5$; $m=0.5 ; r=1.2$.

globally attractive. From Theorem $(1.3)$ we have $\lim \sup u(t) \leq k$. We know that from the second equation that

$$
v_{t}=v\left(-m+e a_{1} \sqrt{(1-P) u}+e a_{2} P u\right) .
$$

It is well known that there exists $T>0$ such that for $t>T$, there exists $\varepsilon>0$ such that $u(t) \leq k+\varepsilon$. Choosing $0<\varepsilon<u^{*}-k$. Thus, we can obtain

$$
v_{t} \leq v\left(-m+e a_{1} \sqrt{(1-P)(k+\varepsilon)}+e a_{2} P(k+\varepsilon)\right),
$$

where

$$
-m+e a_{1} \sqrt{(1-P)(k+\varepsilon)}+e a_{2} P(k+\varepsilon)<0,
$$

which leads to

$$
v(t) \leq v(0) \exp \left[\left(-m+e b_{1} \sqrt{k+\varepsilon}+e a_{2} P(k+\varepsilon)\right) t\right] .
$$

Putting $t \rightarrow+\infty$ we obtain that $v(t) \rightarrow 0$ for $t \rightarrow+\infty$. By replacing this result in the first equation we obtain $u(t) \rightarrow k$ for $t \rightarrow+\infty$. The proof is completed.

Now, let us prove the existence of Hopf bifurcation. Firstly, we consider the carrying capacity k as bifurcation parameter. we calculate the Jacobian matrix of the system (1.3) at the positive equilibrium $\left(u^{*}, v^{*}\right)$ and it is 
given by

$$
J\left(u^{*}, v^{*}\right)=\left(\begin{array}{cc}
a_{11}(k) & a_{12} \\
a_{21}(k) & 0
\end{array}\right),
$$

where

$$
\begin{aligned}
a_{11}(k) & =r\left(1-\frac{2 u^{*}}{k}\right)-\frac{v^{*}}{2 \sqrt{u^{*}}}\left(a_{1} \sqrt{1-P}+2 a_{2} P \sqrt{u^{*}}\right), \\
& =\frac{r a_{1} \sqrt{1-P}}{2\left(a_{1} \sqrt{1-P}+a_{2} P \sqrt{u^{*}}\right)}-\frac{r u^{*}}{k}\left(\frac{2 a_{1} \sqrt{1-P}+a_{2} P \sqrt{u^{*}}}{\left.2 a_{1} \sqrt{1-P}+a_{2} P \sqrt{u^{*}}\right)}\right), \\
a_{21}(k) & =e v^{*}\left(\frac{a_{1} \sqrt{1-P}}{2 \sqrt{u^{*}}}+a_{2} P\right), \\
& =\frac{r e}{2}\left(1+\frac{a_{2} P \sqrt{u^{*}}}{a_{1} \sqrt{(1-P)}+a_{2} P \sqrt{u^{*}}}\right)\left(1-\frac{u^{*}}{k}\right)>0, \\
a_{12} & =-\left(a_{1} \sqrt{1-P} \sqrt{u^{*}}+a_{2} P u^{*}\right)=-\frac{m}{e}<0 .
\end{aligned}
$$

The characteristic equation is given by

$$
\lambda^{2}-T_{0}(k) \lambda+D_{0}=0
$$

where

$$
\begin{aligned}
& T_{0}(k)=\frac{r a_{1} \sqrt{1-P}}{2\left(a_{1} \sqrt{1-P}+a_{2} P \sqrt{u^{*}}\right)}-\frac{r u^{*}}{k}\left(\frac{2 a_{1} \sqrt{1-P}+a_{2} P \sqrt{u^{*}}}{2\left(a_{1} \sqrt{1-P}+a_{2} P \sqrt{u^{*}}\right)}\right), \\
& D_{0}(k)=\frac{r m}{2}\left(1+\frac{a_{2} P \sqrt{u^{*}}}{a_{1} \sqrt{(1-P)}+a_{2} P \sqrt{u^{*}}}\right)\left(1-\frac{u^{*}}{k}\right)>0 .
\end{aligned}
$$

Obviously, $D_{0}(k)>0$ which means that the system (1.3) undergoes a Hopf bifurcation if $T_{0}(k)=0$ and it is equivalent to

$$
k=k^{*}=\frac{u^{*}\left(2 a_{1} \sqrt{1-P}+a_{2} P \sqrt{u^{*}}\right)}{a_{1} \sqrt{1-P}} .
$$

Remark 3.3. It is easy to verify that $k^{*}>u^{*}$ which means that this bifurcation point exists.

Letting $\lambda(k)=\beta(k) \pm \omega(k)$ be the roots of the characteristic equation (3.4) satisfying $\beta\left(k^{*}\right)=0, \omega\left(k^{*}\right)=$ $\sqrt{D_{0}\left(k^{*}\right)}$. Then we have

$$
\beta^{\prime}\left(k^{*}\right)=\frac{1}{2} T_{0}^{\prime}\left(k^{*}\right)=\frac{1}{2} \frac{r u^{*}}{k^{* 2}}\left(\frac{2 a_{1} \sqrt{1-P}+a_{2} P \sqrt{u^{*}}}{2\left(a_{1} \sqrt{1-P}+a_{2} P \sqrt{u^{*}}\right)}\right)>0,
$$

which together with the fact that the characteristic equation (3.4) has a pair of purely imaginary roots $\pm i \sqrt{D_{0}\left(k^{*}\right)}$ at $k^{*}$, implies that the system (1.3) undergoes Hopf bifurcation at $k=k^{*}$.

Thus, we deduce that the interior equilibrium $\left(u^{*}, v^{*}\right)$ is locally asymptotically stable for $k<k^{*}$ and instable for $k>k^{*}$ and the system (1.3) undergoes Hopf bifurcation at $k=k^{*}$.

\section{Stability, Hopf BIfurcation For the Diffusive System}

In this section, the stability of the positive equilibrium $\left(u^{*}, v^{*}\right)$ has been studied. Further, the existence of the Hopf bifurcation for the system (1.4), non existence of diffusion driven instability has been successfully proved. Throughout the rest part of the paper, the condition $k>u^{*}$ has been assumed to be verified. 


\subsection{Characteristic equation}

Consider the following problem

$$
\left\{\begin{array}{c}
-\Delta \psi=\kappa \psi \quad x \in(0, l \pi) \\
\psi_{x}=0, x=0, l \pi
\end{array}\right.
$$

the eigenvalue of the problem $(4.1)$ are $\kappa_{n}=\left(\frac{n}{l}\right)^{2}, n=0,1,2, \ldots$, and the corresponding eigenfunction are

$$
\psi_{n}(x)=\left\{\begin{aligned}
\sqrt{\frac{1}{\pi}}, & n=0, \\
\sqrt{\frac{2}{\pi}} \cos \frac{n}{l} x, \quad n & =1,2,3, \ldots,
\end{aligned}\right.
$$

and $\left\{\psi_{n}(x)\right\}_{1}^{\infty}$ describes the orthogonal basis of $L^{2}(0, l \pi)$. Now letting

$$
\chi=\left\{U=(u, v)^{T} \in W^{2,2}(0, l \pi) / u_{x}=v_{x}=0 \text { at } x=0, l \pi\right\} .
$$

The space $\chi$ is Banach space, and $\Omega=L^{2}(0, l \pi) \times L^{2}(0, l \pi)$ is Hilbert space with the inner product $\left(U_{1}, U_{2}\right)=$ $\int_{0}^{l \pi}\left(u_{1} u_{2}+v_{1} v_{2}\right) \mathrm{d} x$, where $U_{1}=\left(u_{1}, v_{1}\right)^{T}$ and $U_{2}=\left(u_{2}, v_{2}\right)^{T}$. Defining the following mapping $F:(0, \infty) \times \chi \rightarrow \Omega$ by

$$
F(k, U)=\left(\begin{array}{c}
\mathrm{d} u_{x x}+r u\left(1-\frac{u}{k}\right)-a_{1} \sqrt{(1-P) u} v-a_{2} P u v \\
\tilde{\mathrm{d}} v_{x x}-m y+e a_{1} \sqrt{(1-P) u} v+e a_{2} P u v
\end{array}\right)
$$

where $U=(u, v)^{T}$. Then for any $(u, v)^{T} \in \chi$,

$$
U=(u, v)^{T} \text { is solution of }(1.4) \Leftrightarrow F(k, U)=0 .
$$

At the homogeneous steady state $\left(u^{*}, v^{*}\right)$ the Frechet derivative of $F(k, U)$ for $U$ is given as follows

$$
\begin{aligned}
L(k) & =\operatorname{diag}(\mathrm{d} \Delta, \tilde{\mathrm{d}} \Delta)+J\left(u^{*}, v^{*}\right), \\
& =\left(\begin{array}{cc}
\mathrm{d} \Delta u+\frac{r a_{1} \sqrt{1-P}}{2\left(a_{1} \sqrt{1-P}+a_{2} P \sqrt{u^{*}}\right)}-\frac{r u^{*}}{k}\left(\frac{2 a_{1} \sqrt{1-P}+a_{2} P \sqrt{u^{*}}}{2\left(a_{1} \sqrt{1-P}+a_{2} P \sqrt{u^{*}}\right)}\right)-\frac{m}{e} \\
\frac{r e}{2}\left(1+\frac{a_{2} P \sqrt{u^{*}}}{a_{1} \sqrt{(1-P)}+a_{2} P \sqrt{u^{*}}}\right)\left(1-\frac{u^{*}}{k}\right) & \tilde{\mathrm{d}} \Delta
\end{array}\right) .
\end{aligned}
$$

The characteristic equation of $L(k)$ is $L(k)(u, v)=\lambda(u, v)$, (where $(u, v) \in \chi)$ and $\lambda$ is an eigenvalue, and $(u, v)$ verify the following system

$$
\left\{\begin{array}{c}
\mathrm{d} \Delta u+\left[\frac{r a_{1} \sqrt{1-P}}{2\left(a_{1} \sqrt{1-P}+a_{2} P \sqrt{\left.u^{*}\right)}\right.}-\frac{r u^{*}}{k}\left(\frac{2 a_{1} \sqrt{1-P}+a_{2} P \sqrt{u^{*}}}{2\left(a_{1} \sqrt{1-P}+a_{2} P \sqrt{u^{*}}\right)}\right)\right] u-\frac{m}{e} v=\lambda u, \quad x \in(0, l \pi), \\
\tilde{\mathrm{d}} \Delta v+\frac{r e}{2}\left(1+\frac{a_{2} P \sqrt{u^{*}}}{a_{1} \sqrt{(1-P)}+a_{2} P \sqrt{u^{*}}}\right)\left(1-\frac{u^{*}}{k}\right) u=\lambda u, \quad x \in(0, l \pi), \\
u_{x}=v_{x}=0 \quad x=0, l \pi .
\end{array}\right.
$$


Letting $u=\sum_{n=0}^{\infty} a_{n} \psi_{n}$ and $v=\sum_{n=0}^{\infty} b_{n} \psi_{n}$. Then the characteristic equation becomes

$$
\sum_{n=0}^{\infty}\left(\operatorname{diag}\left(-\mathrm{d} \kappa_{n},-\tilde{\mathrm{d}} \kappa_{n}\right)+J\left(E^{*}\right)\right)\left(\begin{array}{c}
a_{n} \\
b_{n}
\end{array}\right) \psi_{n}=0 .
$$

Letting $\left|\operatorname{diag}\left(-\mathrm{d} \kappa_{n},-\tilde{\mathrm{d}} \kappa_{n}\right)+J\left(E^{*}\right)\right|=0, n=0,1,2, \ldots$, we obtain

$$
\lambda^{2}-T_{n}(k) \lambda+D_{n}(k)=0,
$$

with

$$
\begin{aligned}
& T_{n}(k)=\frac{r a_{1} \sqrt{1-P}}{2\left(a_{1} \sqrt{1-P}+a_{2} P \sqrt{u^{*}}\right)}-\frac{r u^{*}}{k}\left(\frac{2 a_{1} \sqrt{1-P}+a_{2} P \sqrt{u^{*}}}{2\left(a_{1} \sqrt{1-P}+a_{2} P \sqrt{u^{*}}\right)}\right)-(d+\tilde{d})\left(\frac{n}{l}\right)^{2}, \\
& D_{n}(k)=d \tilde{d}\left(\frac{n}{l}\right)^{4}-\tilde{d}\left(\frac{n}{l}\right)^{2}\left[\frac{r a_{1} \sqrt{1-P}}{2\left(a_{1} \sqrt{1-P}+a_{2} P \sqrt{u^{*}}\right)}-\frac{r u^{*}}{k}\left(\frac{2 a_{1} \sqrt{1-P}+a_{2} P \sqrt{u^{*}}}{2\left(a_{1} \sqrt{1-P}+a_{2} P \sqrt{u^{*}}\right)}\right)\right] \\
& +\frac{r m}{2}\left(1+\frac{a_{2} P \sqrt{u^{*}}}{a_{1} \sqrt{(1-P)}+a_{2} P \sqrt{u^{*}}}\right)\left(1-\frac{u^{*}}{k}\right) .
\end{aligned}
$$

\subsection{The existence of Hopf bifurcation}

Recall that a Hopf bifurcation occurs if and only if $T_{n}(k)=0$ and $D_{n}(k)>0$. Obviously $D_{0}(k)>0$ and $\lim _{n \rightarrow+\infty} D_{n}(k)=+\infty$. The critical value of the bifurcation parameter $k$ must be the solution of the following equation of the variable $k$

$$
\frac{r a_{1} \sqrt{1-P}}{2\left(a_{1} \sqrt{1-P}+a_{2} P \sqrt{u^{*}}\right)}-\frac{r u^{*}}{k}\left(\frac{2 a_{1} \sqrt{1-P}+a_{2} P \sqrt{u^{*}}}{2\left(a_{1} \sqrt{1-P}+a_{2} P \sqrt{u^{*}}\right)}\right)-(d+\tilde{d})\left(\frac{n}{l}\right)^{2}=0,
$$

which is equivalent to

$$
k=k(n),
$$

where

$$
k(n)=\frac{r u^{*}\left(2 a_{1} \sqrt{1-P}+a_{2} P \sqrt{u^{*}}\right)}{r b_{1}-2(d+\tilde{d})\left(\frac{n}{l}\right)^{2}\left(a_{1} \sqrt{1-P}+a_{2} P \sqrt{u^{*}}\right)} .
$$

Then we have the following results:

Lemma 4.1. Putting

$$
N_{1}=\max \left\{n \in \mathbb{N} / r a_{1} \sqrt{1-P}-2(d+\tilde{d})\left(\frac{n}{l}\right)^{2}\left(a_{1} \sqrt{1-P}+a_{2} P \sqrt{u^{*}}\right)>0\right\} .
$$

Hopf bifurcation occurs for the system (1.4) at $k=k(n)$ and for $n \leq N_{1}$ (where $k(n)$ is defined in (4.10)) and $k(n)$ verifies the following estimation

$$
u^{*}<k(0)<k(1)<. .<k(n)<k(n+1)<\ldots<k\left(N_{1}\right) .
$$

Proof. Hopf bifurcation occurs if and only if $T_{n}(k)=0$, which equivalent to

$$
T_{0}(k)=(d+\tilde{d})\left(\frac{n}{l}\right)^{2},
$$


and it is easy to see that $\lim _{k \rightarrow u^{*}} T_{0}(k)=-r<0$, and

$$
T_{0}^{\prime}(k)=\frac{r}{2 k^{2}}\left(\frac{2 a_{1} \sqrt{1-P}+a_{2} P \sqrt{u^{*}}}{a_{1} \sqrt{1-P}+a_{2} P \sqrt{u^{*}}}\right)>0,
$$

and

$$
\lim _{k \rightarrow+\infty} T_{0}(k)=\frac{r a_{1} \sqrt{1-P}}{2\left(a_{1} \sqrt{1-P}+a_{2} P \sqrt{u^{*}}\right)}>0 .
$$

From (4.13) and (4.14), $T_{0}(k)$ is strictly increasing with respect to the carrying capacity $k$ and the solution of the equation $T_{0}(k)=0$ is

$$
k=k^{*}>u^{*},
$$

where $k^{*}$ is defined by (3.6). The equation (4.13) possesses a solution if and only if the constant integer $n$ verifies

$$
(d+\tilde{d})\left(\frac{n}{l}\right)^{2}<\frac{r a_{1} \sqrt{1-P}}{2\left(a_{1} \sqrt{1-P}+a_{2} P \sqrt{u^{*}}\right)},
$$

which equivalent to $n<N_{1}$.

The function $(d+\tilde{d})\left(\frac{n}{l}\right)^{2}$ is strictly increasing with respect to $n$, which leads to the estimation obtained in (4.12) (see Fig. 7). The proof is completed.

Now we put $\lambda(k)=\beta(k) \pm i \omega(k)$ as the solution of the characteristic equation with $\beta(k(n))=0$ and $\omega(k(n))=\sqrt{D(k(n))}$, then

$$
\beta^{\prime}(k(n))=\frac{r}{2 k^{2}(n)}\left(\frac{2 a_{1} \sqrt{1-P}+a_{2} P \sqrt{u^{*}}}{2\left(a_{1} \sqrt{1-P}+a_{2} P \sqrt{u^{*}}\right)}\right)>0 .
$$

Under the result (4.18) the bifurcation points and their order is given by the following theorem

Theorem 4.2. If there exists $N^{*} \leq N_{1}$ a critical value such that $i_{0}=0<i_{1}<\ldots<i_{N^{*}} \leq N_{1}$ and $D_{i \xi}(k(i \xi))>$ $0, \xi=0 \ldots N^{*}$ we have the following estimation:

$$
u^{*}<k(0)<k(1)<. .<k(n)<k(n+1)<\ldots<k\left(i_{N^{*}}\right) .
$$

In order to the above analysis we have the following theorem

Theorem 4.3. Assume that $k>u^{*}$ holds, then we have the following results

(i) If $n=0$ the equilibrium $\left(u^{*}, v^{*}\right)$ is asymptotically stable for $0<k<k^{*}:=k(0)$ and unstable for $k>k^{*}$.

(ii) The system (1.4) has a Hopf bifurcation near $\left(u^{*}, v^{*}\right)$ for $k=k(n)$ where a spatially homogenous periodic solution appears for $n=0$, and spatially nonhomogeneous periodic solution for $n=1,2, \ldots, i_{N^{*}}$.

Remark 4.4. For Theorem 4.2 we have $T_{n}=0$ for $k=k_{n}, n=0,1, \ldots, i_{N^{*}}$ and it is well known that $D_{0}>0$ for $k>u^{*}$. Thus there exist an integer denoted by $N^{*}$ such that $D_{n}>0$ for $n=0,1, \ldots, N^{*}$. Taking $i_{N^{*}}=$ $\min \left\{N_{1}, N^{*}\right\}$. Thus we have:

(i) $T_{n}<0$ for $n=0,1, \ldots, i_{N^{*}}$.

(ii) $D_{n}>0$ for $n=0,1, \ldots, i_{N^{*}}$.

(iii) The transversality condition (4.18).

Thus the system undergoes Hopf bifurcation at $k=k(n)$ for $n=0,1, \ldots, i_{N^{*}}$. 


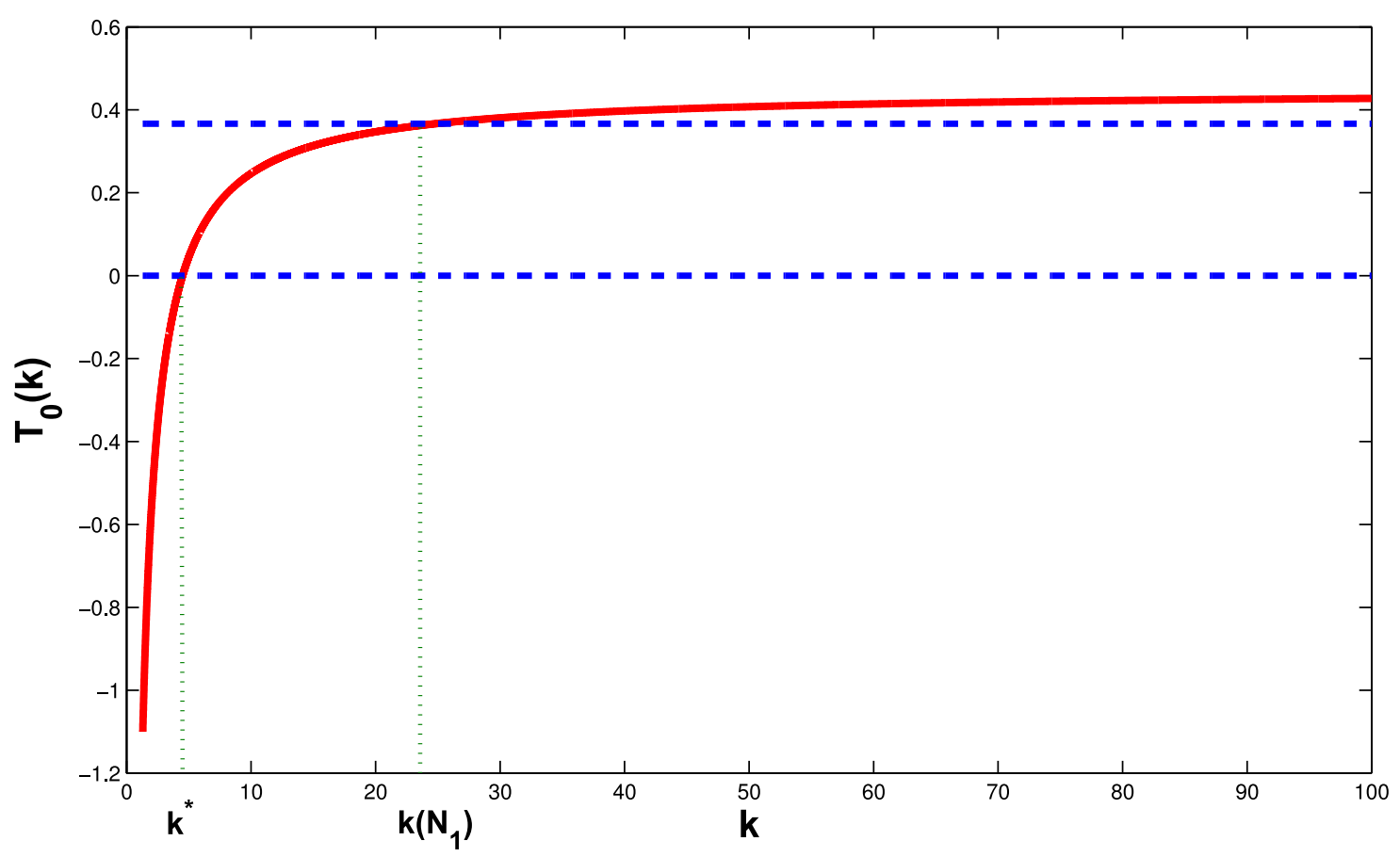

Figure 7 . The existence and the order of Hopf bifurcation points the solution of the equation (4.13) where $a=3.5 ; P=0.7 ; l=5 ; e=0.5 ; m=0.5 ; r=2.2 ; d=0.02 ; \widetilde{d}=0.1$ which means $u^{*}=1.3005 ; N_{1}=6$.

\subsection{Non existence of Turing instability}

In this subsection, our main focus is on proving that the system (1.4) can not undergo diffusion driven instability. Note that Turing driven instability occurs when the equilibrium point is linearly stable in the absence of spatial diffusion and in the presence of this last becomes unstable, which means that we will assume that $u^{*}<k<k^{*}$.

Lemma 4.5. The system (1.4) has no Turing instability at $\left(u^{*}, v^{*}\right)$.

Proof. Obviously $D_{0}(k)>0$, then for having the existence of Turing instability we must prove $D_{n}(k)<0$ which can be rewritten as follows

$$
D_{n}(k)=D\left(\left(\frac{n}{l}\right)^{2}\right)=d \tilde{d}\left(\left(\frac{n}{l}\right)^{2}\right)^{2}-\tilde{d}\left(\frac{n}{l}\right)^{2}\left[\frac{r a_{1} \sqrt{1-P}}{2\left(a_{1} \sqrt{1-P}+a_{2} P \sqrt{u^{*}}\right)}-\frac{r u^{*}}{k}\left(\frac{2 a_{1} \sqrt{1-P}+a_{2} P \sqrt{u^{*}}}{2\left(a_{1} \sqrt{1-P}+a_{2} P \sqrt{u^{*}}\right)}\right)\right]+D_{0}(k) .
$$

Thus, using the fact that $u^{*}<k<k^{*}$, we obtain that

$$
\frac{r a_{1} \sqrt{1-P}}{2\left(a_{1} \sqrt{1-P}+a_{2} P \sqrt{u^{*}}\right)}-\frac{r u^{*}}{k}\left(\frac{2 a_{1} \sqrt{1-P}+a_{2} P \sqrt{u^{*}}}{2\left(a_{1} \sqrt{1-P}+a_{2} P \sqrt{u^{*}}\right)}\right)<0
$$

which means $D_{n}(k)>0$ for any integer $\mathrm{n}$ the we deduce the non existence of Turing driven instability. The proof is completed.

\subsection{Direction and stability of Hopf bifurcation}

In this section our aim is to study the stability of the homogeneous and the non homogeneous periodic solution using the normal form on the center manifold at the Hopf bifurcation points. Before that we will make 
a change of variable by putting $\tilde{u}=u-u^{*}$ and $\tilde{v}=v-v^{*}$ where $v^{*}=v_{\mu}^{*}=\frac{r \sqrt{u^{*}}}{a_{1} \sqrt{1-P}+a_{2} P \sqrt{u^{*}}}\left(1-\frac{u^{*}}{\tilde{k}+\mu}\right)$ and $\mu=k-k(n)$ where $\mu=0$ is equivalent to and $k=k(n), n=1,2, \ldots, i_{N^{*}}$ and dropping tilde for convenience, the system (1.4) becomes:

$$
\left\{\begin{array}{l}
u_{t}-\mathrm{d} u_{x x}=r\left(u+u^{*}\right)\left(1-\frac{\left(u+u^{*}\right)}{k(n)+\mu}\right)-a_{1} \sqrt{(1-P)\left(u+u^{*}\right)} v-a_{2} P\left(u+u^{*}\right)\left(v+v^{*}\right), \\
v_{t}-\tilde{\mathrm{d}} v_{x x}=-m y+e a_{1} \sqrt{(1-P)\left(u+u^{*}\right)}\left(v+v^{*}\right)+e a_{2} P\left(u+u^{*}\right)\left(v+v^{*}\right),
\end{array} \quad x \in[0, l \pi] ; t>0\right.
$$

and it is equivalent to

$$
\dot{U}(t)=L U+F(U, \mu)
$$

where

$$
L=D \Delta+J\left(E^{*}\right)=\left(\begin{array}{cc}
a_{11}(\mu)+\mathrm{d} \frac{\partial^{2}}{\partial x^{2}} & a_{12} \\
a_{21}(\mu) & \tilde{\mathrm{d}} \frac{\partial^{2}}{\partial x^{2}}
\end{array}\right),
$$

and

$$
\begin{gathered}
F\left(U, R^{*}\right)=\left(\begin{array}{c}
F_{1}(U, \mu) \\
F_{2}(U, \mu)
\end{array}\right), \\
=\left(\begin{array}{c}
r\left(u+u^{*}\right)\left(1-\frac{\left(u+u^{*}\right)}{k(n)+\mu}\right)-a_{1} \sqrt{(1-P)\left(u+u^{*}\right)} v-a_{2} P\left(u+u^{*}\right)\left(v+v^{*}\right)-a_{11}(\mu) u-a_{12} v \\
-m y+e a_{1} \sqrt{(1-P)\left(u+u^{*}\right)}\left(v+v^{*}\right)+e a_{2}\left(u+u^{*}\right)\left(v+v^{*}\right)-a_{21}(\mu) u
\end{array}\right),
\end{gathered}
$$

where $a_{11}(\mu), a_{12}, a_{21}(\mu)$ are defined in (3.3). Now we define the following complex space of $\chi$ by the decomposition:

$$
\chi_{\mathbb{C}}=\chi \oplus i \chi
$$

and the inner product

$$
\left\langle U_{1}, U_{2}\right\rangle=\int_{0}^{\pi} \bar{u}_{1} u_{2}+\bar{v}_{1} v_{2} \mathrm{~d} x \quad \text { where } U_{1}=\left(u_{1}, v_{1}\right)^{T}, U_{2}=\left(u_{2}, v_{2}\right)^{T} \in \chi_{\mathbb{C}} .
$$

The adjoint operator $L^{*}$ is given by

$$
L^{*}=D \Delta+J^{*}(\mu)=\left(\begin{array}{cc}
a_{11}(\mu)+\mathrm{d} \frac{\partial^{2}}{\partial x^{2}} & a_{21}(\mu) \\
a_{12} & \tilde{\mathrm{d}} \frac{\partial^{2}}{\partial x^{2}}
\end{array}\right) .
$$

Then $L_{n}^{*}(\mu)=-n^{2} D+J^{*}(\mu)$ where $J^{*}(\mu)$ is the adjoint matrix of $J(\mu)$. Now we define the eigenfunction of the $L_{n}(\mu)$ and $L_{n}^{*}(\mu)$ corresponding the eigenvalue $i \omega$ and $-i \omega$ by

$$
q=\left(\begin{array}{l}
a_{n} \\
b_{n}
\end{array}\right) \cos \left(\frac{n}{l} x\right) \text { and } q^{*}=\left(\begin{array}{l}
a_{n}^{*} \\
b_{n}^{*}
\end{array}\right) \cos \left(\frac{n}{l} x\right)
$$


such that $\left\langle q^{*}, q\right\rangle=1$ and $\left\langle q^{*}, \bar{q}\right\rangle=0$ and also $L_{n}^{*}(\mu) q^{*}=-i \omega q^{*}$. Where

$$
\left(\begin{array}{l}
a_{n} \\
b_{n}
\end{array}\right)=\frac{\left(i \omega-\left(a_{11}-\mathrm{d}\left(\frac{n}{l}\right)^{2}\right.\right.}{a_{21}} n=0,1, \ldots, i_{N^{*}} \text { and } \mu=0 .
$$

And for $\mu=0$ we have

$$
\left(\begin{array}{l}
a_{n}^{*} \\
b_{n}^{*}
\end{array}\right)=\left\{\begin{array}{l}
\left(\frac{1}{l \pi} \frac{a_{21} a_{12}}{a_{21} a_{12}+\left(i \omega+a_{11}\right)^{2}}, \frac{1}{l \pi} \frac{-a_{12}\left(i \omega+a_{11}\right)}{a_{21} a_{12}+\left(i \omega+a_{11}\right)^{2}}\right)^{T} \quad \text { if } n=0, \\
\left(\frac{2}{l \pi} \frac{a_{21} a_{12}}{a_{21} a_{12}+\left(i \omega+a_{11}-\mathrm{d}\left(\frac{n}{l}\right)^{2}\right)^{2}}, \frac{2}{l \pi} \frac{-a_{21} a_{12}}{a_{21} a_{12}+\left(i \omega+a_{11}-\mathrm{d}\left(\frac{n}{l}\right)^{2}\right)^{2}}\right)^{T} \quad \text { if } n \neq 0
\end{array} .\right.
$$

and use the decomposition of our space

$$
\chi=\chi^{\mathbb{C}} \oplus \chi^{s},
$$

where $\chi^{\mathbb{C}}:=\{z q+\bar{z} \bar{q} / z \in \mathbb{C}\}$ and $\chi^{s}:=\left\{U \in \chi /\left\langle q^{*}, U\right\rangle=0\right\}$ for any $U=(R, P)^{T} \in \chi$ there exists $z \in \mathbb{C}$ $\left(u \in \chi^{\mathbb{C}}\right.$ means there exist $z \in \mathbb{C}$ such that $\left.u=z q+\bar{z} \bar{q}\right)$ and $w=\left(w_{1}, w_{2}\right)^{T} \in \chi^{s}$ such that

$$
U=z q+\bar{z} \bar{q}+w=\left\{\begin{array}{c}
R=a_{n} z \cos (n x)+\bar{a}_{n} \bar{z} \cos (n x)+w_{1}, \\
P=b_{n} z \cos (n x)+\bar{b}_{n} \bar{z} \cos (n x)+w_{1} .
\end{array}\right.
$$

Now, we will follow the steps given in [12] so we have

$$
\left\{\begin{array}{c}
\frac{\mathrm{d} z}{\mathrm{~d} t}=i \omega z+\left\langle q^{*}, F_{n}(U)\right\rangle \\
\frac{\mathrm{d} w}{\mathrm{~d} t}=L(\mu) w+H(z, \bar{z}, w)
\end{array}\right.
$$

where

$$
\left\{\begin{array}{l}
H(z, \bar{z}, w)=F_{n}(U)-\left\langle q^{*}, F_{n}(U)\right\rangle q-\left\langle\bar{q}^{*}, F_{n}(U)\right\rangle \bar{q} \\
F_{n}(U)=\frac{1}{2} Q_{U U}+\frac{1}{6} C_{U U U}+o\left(|U|^{4}\right)
\end{array}\right.
$$

where $Q_{U U}$ and $C_{U U U}$ are the second and the third order of the system (4.8) then we have

$$
\left\{\begin{array}{l}
Q_{q q}=\left(\begin{array}{c}
c_{n} \\
d_{n}
\end{array}\right) \cos ^{2}\left(\frac{n}{l} x\right), \\
Q_{\bar{q} \bar{q}}=\bar{Q}_{q q}, \\
Q_{q \bar{q}}=\left(\begin{array}{c}
e_{n} \\
f_{n}
\end{array}\right) \cos ^{2}\left(\frac{n}{l} x\right), \\
Q_{q \bar{q}}=\left(\begin{array}{c}
g_{n} \\
h_{n}
\end{array}\right) \cos ^{2}\left(\frac{n}{l} x\right),
\end{array}\right.
$$


where the partial derivative evaluated at origin

$$
\left\{\begin{array}{l}
c_{n}=F_{1 R R} a_{n}^{2}+2 F_{1 R P} a_{n} b_{n}+F_{1 P P} b_{n}^{2}, \\
d_{n}=F_{2 R R} a_{n}^{2}+2 F_{2 R P} a_{n} b_{n}+F_{2 P P} b_{n}^{2}, \\
e_{n}=F_{1 R R} a_{n} \bar{a}_{n}+F_{1 R P}\left(a_{n} \bar{b}_{n}+\bar{a}_{n} b_{n}\right)+F_{1 P P} b_{n} \bar{b}_{n}, \\
f_{n}=F_{2 R R} a_{n} \bar{a}_{n}+F_{2 R P}\left(a_{n} \bar{b}_{n}+\bar{a}_{n} b_{n}\right)+F_{2 P P} b_{n} \bar{b}_{n}, \\
g_{n}=F_{1 R R R} a_{n}^{2} \bar{a}_{n}+F_{1 R R P}\left(a_{n}^{2} \bar{b}_{n}+2 a_{n} \bar{a}_{n} b_{n}\right)+F_{1 R P P}\left(\bar{a}_{n} b_{n}^{2}+2 a_{n} b_{n} \bar{b}_{n}\right)+F_{1 P P P} b_{n}^{2} \bar{b}_{n}, \\
h_{n}=F_{2 R R R} a_{n}^{2} \bar{a}_{n}+F_{2 R R P}\left(a_{n}^{2} \bar{b}_{n}+2 a_{n} \bar{a}_{n} b_{n}\right)+F_{2 R P P}\left(\bar{a}_{n} b_{n}^{2}+2 a_{n} b_{n} \bar{b}_{n}\right)+F_{2 P P P} b_{n}^{2} \bar{b}_{n} .
\end{array}\right.
$$

Then, the reaction-diffusion system restricted to the center of manifold is given by

$$
\frac{\mathrm{d} z}{\mathrm{~d} t}=i \omega z+\left\langle q^{*}, F_{n}(U)\right\rangle=i \omega z+\sum_{2 \leq i+j \leq 3} \frac{g_{i j}}{i ! j !} z^{i} z^{j}+o\left(|z|^{4}\right),
$$

where

$$
\left\{\begin{array}{l}
g_{20}=\left\langle q^{*}, Q_{q q}\right\rangle, \\
g_{11}=\left\langle q^{*}, Q_{q \bar{q}}\right\rangle, \\
g_{02}=\left\langle q^{*}, Q_{\bar{q} \bar{q}}\right\rangle, \\
g_{21}=2\left\langle q^{*}, Q_{w_{11} q}\right\rangle+\left\langle q^{*}, Q_{w_{20} \bar{q}}\right\rangle+\left\langle q^{*}, C_{q q \bar{q}}\right\rangle .
\end{array}\right.
$$

Then we have two cases:

(i) If $n=0$ (for the spatially homogenous Hopf bifurcation)

$$
\left\{\begin{array}{l}
g_{20}=l \pi\left(\bar{a}_{0}^{*} c_{0}+\bar{b}_{0}^{*} d_{0}\right), \\
g_{11}=l \pi\left(\bar{a}_{0}^{*} e_{0}+\bar{b}_{0}^{*} f_{0}\right), \\
g_{02}=l \pi\left(\bar{a}_{0}^{*} \bar{c}_{0}+\bar{b}_{0}^{*} \bar{d}_{0}\right), \\
g_{21}=2\left\langle q^{*}, Q_{w_{11} q}\right\rangle+\left\langle q^{*}, Q_{w_{20} \bar{q}}\right\rangle+\left\langle q^{*}, C_{q q \bar{q}}\right\rangle .
\end{array}\right.
$$

where

$$
\begin{aligned}
&\left\langle q^{*}, Q_{w_{11} q}\right\rangle= l \pi\left[\bar{a}_{0}^{*}\left(F_{1 R R} w_{11}^{(1)} a_{0}+F_{1 R P}\left(w_{11}^{(1)} b_{0}+w_{11}^{(2)} a_{0}\right)+F_{1 P P} w_{11}^{(2)} b_{0}\right)\right. \\
&\left.+\bar{b}_{0}^{*}\left(F_{2 R R} w_{11}^{(1)} a_{0}+F_{2 R P}\left(w_{11}^{(1)} b_{0}+w_{11}^{(2)} a_{0}\right)+F_{2 P P} w_{11}^{(2)} b_{0}\right)\right] . \\
&\left\langle q^{*}, Q_{w_{20}} \bar{q}\right\rangle= l \pi\left[\bar{a}_{0}^{*}\left(F_{1 R R} w_{20}^{(1)} \bar{a}_{0}+F_{1 R P}\left(w_{20}^{(1)} \bar{b}_{0}+w_{20}^{(2)} \bar{a}_{0}\right)+F_{1 P P} w_{20}^{(2)} \bar{b}_{0}\right)\right. \\
&+ \bar{b}_{0}^{*}\left(F_{2 R R} w_{20}^{(1)} \bar{a}_{0}+F_{2 R P}\left(w_{20}^{(1)} \bar{b}_{0}+w_{20}^{(2)} \bar{a}_{0}\right)+F_{2 P P} w_{20}^{(2)} \bar{b}_{0}\right) . \\
&\left\langle q^{*}, C_{q q \bar{q}}\right\rangle=l \pi\left(\bar{a}_{0}^{*} g_{0}+\bar{b}_{0}^{*} h_{0}\right) .
\end{aligned}
$$

and

$$
w_{11}=-\left[L_{0}(\mu)\right]^{-1} H_{11}=\left(\begin{array}{c}
w_{11}^{(1)} \\
w_{11}^{(2)}
\end{array}\right)=\left(\begin{array}{c}
-\frac{H_{11}^{(2)}}{a_{21}} \\
-\frac{H_{11}^{(1)} a_{21}+H_{11}^{(2)} a_{11}}{a_{21} a_{12}}
\end{array}\right) .
$$




$$
w_{20}=\left[2 i \omega \operatorname{diag}(1,1)-L_{0}(\mu)\right]^{-1} H_{20}=\left(\begin{array}{c}
w_{20}^{(1)} \\
w_{20}^{(2)}
\end{array}\right)=\left(\begin{array}{c}
\frac{H_{20}^{(1)} 2 i \omega+H_{20}^{(2)} a_{12}}{\left(2 i \omega-a_{11}\right) 2 i \omega-a_{21} a_{12}} \\
-\frac{H_{20}^{(1)} a_{21}+H_{20}^{(2)}\left(2 i \omega-a_{11}\right)}{\left(2 i \omega-a_{11}\right) 2 i \omega-a_{21} a_{12}}
\end{array}\right) .
$$

where

$$
\begin{aligned}
H_{11} & =Q_{q \bar{q}}-\left\langle q^{*}, Q_{q \bar{q}}\right\rangle q-\left\langle\bar{q}^{*}, Q_{q \bar{q}}\right\rangle \bar{q}=\left(\begin{array}{c}
H_{11}^{(1)} \\
H_{11}^{(2)}
\end{array}\right), \\
& =\left(\begin{array}{c}
e_{0}-l \pi a_{0}\left(\bar{a}_{0}^{*} e_{0}+\bar{b}_{0}^{*} f_{0}\right)-l \pi \bar{a}_{0}\left(a_{0}^{*} e_{0}+b_{0}^{*} f_{0}\right) \\
f_{0}-l \pi b_{0}\left(\bar{a}_{0}^{*} e_{0}+\bar{b}_{0}^{*} f_{0}\right)-l \pi \bar{b}_{0}\left(a_{0}^{*} e_{0}+b_{0}^{*} f_{0}\right)
\end{array}\right) . \\
H_{20} & =Q_{q q}-\left\langle q^{*}, Q_{q q}\right\rangle q-\left\langle\bar{q}^{*}, Q_{q q}\right\rangle \bar{q}=\left(\begin{array}{c}
H_{20}^{(1)} \\
H_{20}^{(2)}
\end{array}\right), \\
& =\left(\begin{array}{c}
c_{0}-\pi a_{0}\left(\bar{a}_{0}^{*} c_{0}+\bar{b}_{0}^{*} d_{0}\right)-\pi \bar{a}_{0}\left(a_{0}^{*} c_{0}+b_{0}^{*} d_{0}\right) \\
d_{0}-\pi b_{0}\left(\bar{a}_{0}^{*} c_{0}+\bar{b}_{0}^{*} d_{0}\right)-\pi \bar{b}_{0}\left(a_{0}^{*} c_{0}+b_{0}^{*} d_{0}\right)
\end{array}\right) .
\end{aligned}
$$

(ii) If $n=1,2, \ldots, i_{N^{*}}$ (for the spatially inhomogeneous Hopf bifurcation)

Not that

$$
\int_{0}^{l \pi} \cos ^{3} \frac{n}{l} x \mathrm{~d} x=0
$$

By straight forward calculation we have

$$
\left.\left.\left\langle q^{*}, Q_{q \bar{q}}\right\rangle=\left\langle q^{*}, Q_{q q}\right\rangle=<\bar{q}^{*}, Q_{q q}\right\rangle=<\bar{q}^{*}, Q_{\bar{q} \bar{q}}\right\rangle=0 .
$$

Then we have

$$
\begin{aligned}
& g_{20}=g_{11}=g_{02}=0, \\
& g_{21}=2\left\langle q^{*}, Q_{w_{11} q}\right\rangle+\left\langle q^{*}, Q_{w_{20} \bar{q}}\right\rangle+\left\langle q^{*}, C_{q q \bar{q}}\right\rangle .
\end{aligned}
$$

where

$$
\begin{gathered}
w_{11}=-\frac{1}{2}\left[L_{n}(\mu)\right]^{-1}\left[\cos \frac{2 n}{l} x+1\right]\left(\begin{array}{l}
e_{n} \\
f_{n}
\end{array}\right), \\
=w_{11 n} \cos \frac{2 n}{l} x+w_{11 n}
\end{gathered}
$$

and

$$
\begin{aligned}
w_{20} & =-\frac{1}{2}\left[2 i \omega \operatorname{diag}(1,1)-L_{n}(\mu)\right]^{-1}\left[\cos \frac{2 n}{l} x+1\right]\left(\begin{array}{l}
c_{n} \\
d_{n}
\end{array}\right), \\
& =w_{20 n} \cos \frac{2 n}{l} x+w_{20 n}
\end{aligned}
$$


Note that $\int_{0}^{l \pi} \cos \frac{2 n}{l} x \cos ^{2} \frac{n}{l} x \mathrm{~d} x=\frac{\pi}{4}$ and $\int_{0}^{l \pi} \cos ^{2} \frac{n}{l} x \mathrm{~d} x=\frac{\pi}{2}$ then we have

$$
\begin{gathered}
\left\langle q^{*}, Q_{w_{11} q}\right\rangle=\frac{3 l \pi}{4}\left[\bar{a}_{n}^{*}\left(F_{1 R R} w_{11 n}^{(1)} a_{n}+F_{1 R P}\left(w_{11 n}^{(1)} b_{n}+w_{11}^{(2)} a_{n}\right)+F_{1 P P} w_{11 n}^{(2)} b_{n}\right) \mid\right. \\
\left.+\bar{b}_{n}^{*}\left(F_{2 R R} w_{11 n}^{(1)} a_{n}+F_{2 R P}\left(w_{11 n}^{(1)} b_{n}+w_{11 n}^{(2)} a_{n}\right)+F_{2 P P} w_{11 n}^{(2)} b_{n}\right)\right] \\
\left\langle q^{*}, Q_{w_{20} \bar{q}}\right\rangle= \\
\frac{3 l \pi}{4}\left[\bar{a}_{0}^{*}\left(F_{1 R R} w_{20 n}^{(1)} \bar{a}_{0}+F_{1 R P}\left(w_{20 n}^{(1)} \bar{b}_{0}+w_{20}^{(2)} \bar{a}_{0}\right)+F_{1 P P} w_{20 n}^{(2)} \bar{b}_{0}\right)\right. \\
+\bar{b}_{0}^{*}\left(F_{2 R R} w_{20 n}^{(1)} \bar{a}_{0}+F_{2 R P}\left(w_{20 n}^{(1)} \bar{b}_{0}+w_{20}^{(2)} \bar{a}_{0}\right)+F_{2 P P} w_{20 n}^{(2)} \bar{b}_{0}\right) \\
\left\langle q^{*}, C_{q q \bar{q}}\right\rangle=\frac{3 l \pi}{8}\left(\bar{a}_{0}^{*} g_{0}+\bar{b}_{0}^{*} h_{0}\right),
\end{gathered}
$$

where

$$
\begin{aligned}
w_{20 n} & =\frac{1}{2}\left[2 i \omega \operatorname{diag}(1,1)-L_{n}(\mu)\right]^{-1}\left(\begin{array}{c}
c_{n} \\
d_{n}
\end{array}\right)=\left(\begin{array}{c}
w_{20 n}^{(1)} \\
w_{20 n}^{(2)}
\end{array}\right), \\
& =\frac{1}{2 \tilde{D}_{n \omega}}\left(\begin{array}{c}
c_{n}\left(2 i \omega+\tilde{d}\left(\frac{n}{l}\right)^{2}\right)+d_{n} a_{12} \\
d_{n}\left(2 i \omega-a_{11}+d\left(\frac{n}{l}\right)^{2}\right)+e_{n} a_{21}
\end{array}\right) .
\end{aligned}
$$

Such that

$$
\begin{aligned}
& \tilde{D}_{n \omega}=\operatorname{det}\left(2 i \omega \operatorname{diag}(1,1)-L_{n}(\mu)\right), \\
& =\left(2 i \omega+\tilde{d}\left(\frac{n}{l}\right)^{2}\right)\left(2 i \omega-a_{11}+d\left(\frac{n}{l}\right)^{2}\right)-a_{12} a_{21},
\end{aligned}
$$

and

$$
\begin{gathered}
w_{11 n}=-\frac{1}{2}\left[L_{n}(\mu)\right]^{-1}\left(\begin{array}{c}
e_{n} \\
f_{n}
\end{array}\right)=\left(\begin{array}{c}
w_{11 n}^{(1)} \\
w_{11 n}^{(2)}
\end{array}\right), \\
=-\frac{1}{2 D_{n}}\left(\begin{array}{c}
-e_{n} \tilde{d}\left(\frac{n}{l}\right)^{2}+f_{n} a_{12} \\
f_{n}\left(a_{11}-d\left(\frac{n}{l}\right)^{2}\right)-e_{n} a_{21}
\end{array}\right),
\end{gathered}
$$

where $D_{n}$ is defined in (4.8) Using the results in Hassard [12] the direction and stability of Hopf bifurcation can be determined by the following values

$$
\begin{gathered}
\mu_{2}=-\frac{\operatorname{Re}\left(c_{1}(0)\right)}{\beta^{\prime}(0)}, \\
v_{2}=2 \operatorname{Re}\left(c_{1}(0)\right),
\end{gathered}
$$

with

$$
c_{1}(0)=\frac{i}{2 \omega}\left(g_{20} g_{11}-2 g_{11} \bar{g}_{11}-\frac{1}{3} g_{02} \bar{g}_{02}\right)+\frac{g_{21}}{2} .
$$



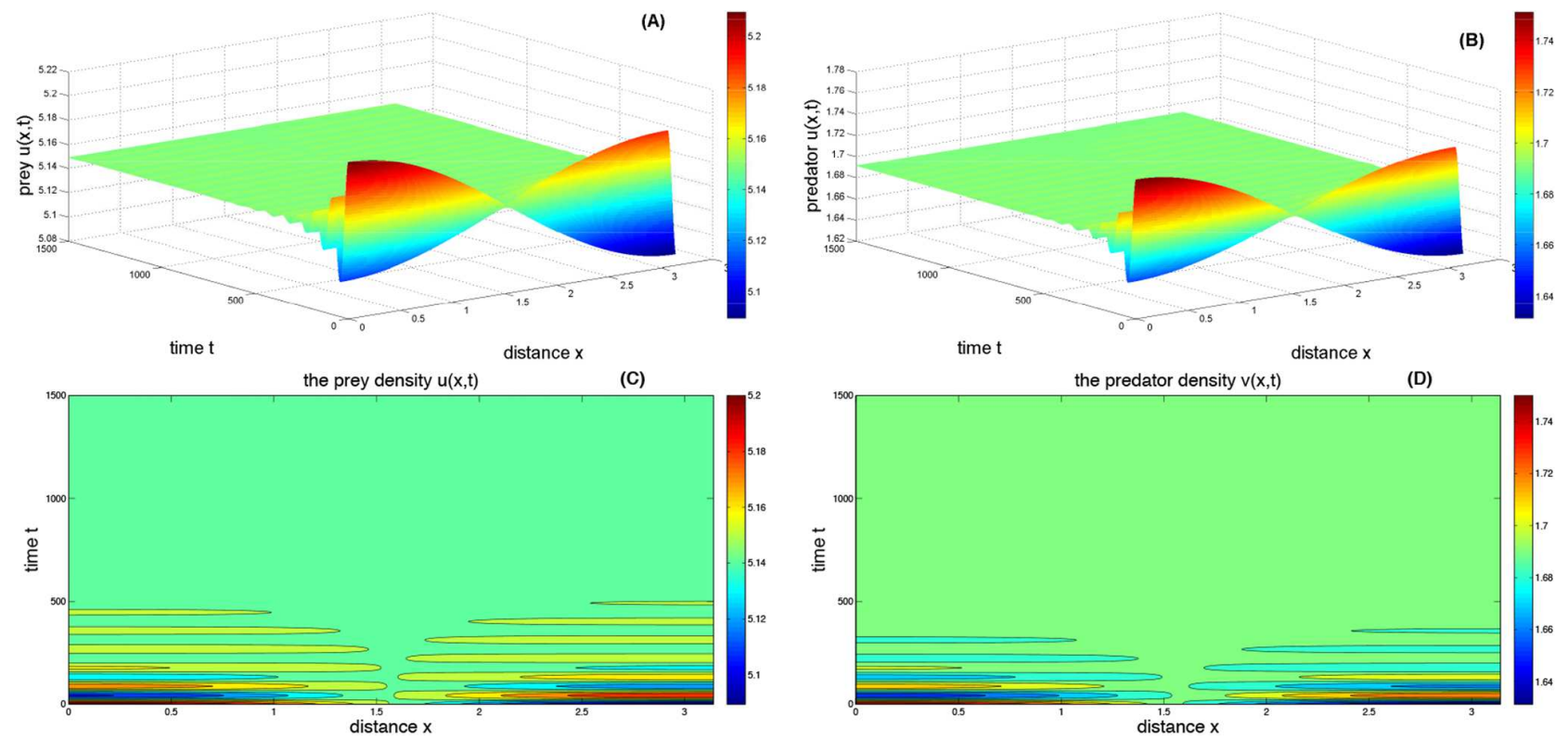

Figure 8. Numerical simulation of the system (1.4) when the equilibrium $\left(u^{*}, v^{*}\right)=$ $(5.1494,1.6639)$ is locally asymptotically stable for $k=32$ and $d=0.02 ; \widetilde{d}=0.01 ; a_{1}=0.5$; $a_{2}=0.7 ; P=0.5 ; l=1 e=0.5 ; m=0.5 ; r=1.2$ with the initial condition $u(0, x)=u^{*}+$ $0.06 \cos x$ and $v(0, x)=v^{*}+0.06 \cos x$.

The direction of Hopf bifurcation is determined by $\mu_{2}$ where the Hopf bifurcation is supercritical (resp. subcritical) if $\mu_{2}>0$ (resp. $\mu_{2}<0$ ). Further, the bifurcating periodic solutions exists for $\mu>0$ (resp. $\mu<0$ ) and the stability of the periodic solutions determined by $v_{2}$, where the bifurcating periodic solution are asymptotically stable (resp. unstable) if $v_{2}<0$ (resp. $\left.v_{2}>0\right)$

\section{NUMERICAL SIMULATION}

In this section we shall discuss the relationship between the theoretical results and the graphical representation, which is given in the following form

Figure 1: Represents the negative effect of the prey escaping rate $P$ on the prey density equilibrium, which has been shown in (2.1). This result shows that the escaping never serves the prey population.

Figure 2: represents the impact of the prey escaping rate $P$ on the predator density equilibrium obtained in Section 2 where it varies between a positive and negative impact which depends only on two critical values $k_{c r}:=\frac{2 m}{e a_{2}}$ and $P_{c r}$. This results shows that the prey escaping can serve (or not) the predator population.

Figure 3: Shows the impact of both the prey escaping rate and the carrying capacity of the environment for the prey population on the predator density equilibrium, which generalizes the obtained figure in Fig. 2. This results can be seen easily from Section 2 . Means that both the carrying capacity of the environment for the prey population and the prey escaping from the pack affect hugely the predator population density equilibrium.

Figure 4: Shows the global stability of the semi trivial equilibrium $(k, 0)$ for $u^{*}>2$ for multi values of the initial conditions. This result has been used to illustrate the results of theorem 3.2, also shows that the predator population extinct for the smaller capacities of the environment for the prey.

Figure 5: Represents the local stability of the interior equilibrium $\left(u^{*}, v^{*}\right)$ for $k<k^{*}$ determined by studying quality of solution of the characteristic equation (3.4). 

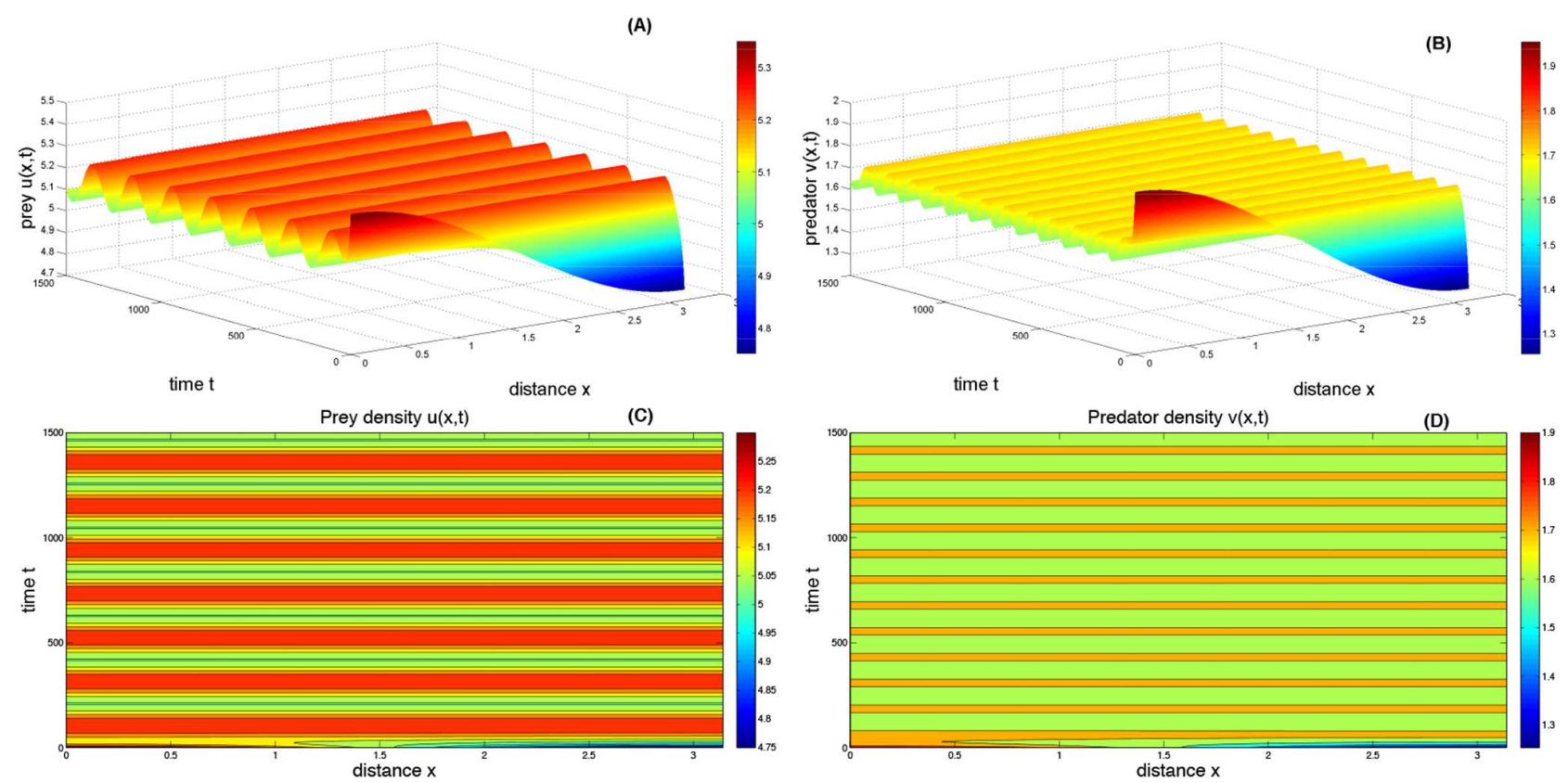

Figure 9. Numerical simulation of the system (1.4) when the equilibrium $\left(u^{*}, v^{*}\right)=$ $(5.1494,1.6827)$ is unstable and the existence of a homogeneous periodic solution for the values $k=34$ and $d=0.02 ; \widetilde{d}=0.01 ; a_{1}=0.5 ; a_{2}=0.7 ; P=0.5 ; l=1 e=0.5 ; m=0.5 ; r=1.2$ with the initial condition $u(0, x)=u^{*}-0.1+0.03 \cos x$ and $v(0, x)=v^{*}-0.06+0.35 \cos x$.

Figure 6: represents that the interior equilibrium is unstable for $k>k^{*}$ and the existence of a stable periodic orbit. This result has been obtained due to the qualitative analysis of the characteristic equation (3.4).

Figure 7: Shows the existence and the order of Hopf bifurcation points which insures the obtained results in theorem 4.2. Further, shows the maximal value of the integer $\mathrm{n}$ in which the system undergoes Hopf bifurcation (in this case we have $N_{1}=6$ ). Also, means that we have seven Hopf bifurcation points.

Now lets investigate with the graphical representation of solutions of the system (1.4), the following numerical schema is used

$$
\left\{\begin{array}{l}
u_{i}^{n+1}=u_{i}^{n}-\mathrm{d} t \frac{\mathrm{d}}{\mathrm{d} x^{2}}\left(2 u_{i}^{n}-u_{i-1}^{n}-u_{i+1}^{n}\right)+f\left(u_{i}^{n}, v_{i}^{n}\right), i=1,2, \ldots, M, n=1,2, \ldots, N, \\
v_{i}^{n+1}=v_{i}^{n}-\mathrm{d} t \frac{\widetilde{\mathrm{d}}}{\mathrm{d} x^{2}}\left(2 v_{i}^{n}-v_{i-1}^{n}-v_{i+1}^{n}\right)+g\left(u_{i}^{n}, v_{i}^{n}\right), i=1,2, \ldots, M, n=1,2, \ldots, N, \\
u_{1}^{n}=u_{0}^{n}, v_{1}^{n}=v_{0}^{n}, u_{M}^{n}=u_{M-1}^{n}, v_{M}^{n}=v_{M-1}^{n}, n=1,2, \ldots, N, \\
u_{i}^{0}=\phi_{i}, v_{i}^{0}=\psi_{i}, i=1,2, \ldots, M,
\end{array}\right.
$$

where $\mathrm{d} x=0.005$ and $\mathrm{d} t=0.05$ and $\phi_{i}=\phi(i \times \mathrm{d} x), \psi_{i}=\psi(i \times \mathrm{d} x), M=\frac{l \pi}{\mathrm{d} x}, N=\frac{T}{\mathrm{~d} t}$ and

$$
\begin{gathered}
f\left(u_{i}^{n}, v_{i}^{n}\right)=r u_{i}^{n}\left(1-\frac{u_{i}^{n}}{k}\right)-a_{1} \sqrt{(1-P) u_{i}^{n}} v_{i}^{n}-a_{2} P u_{i}^{n} v_{i}^{n}, \\
g\left(u_{i}^{n}, v_{i}^{n}\right)=-m v_{i}^{n}+e a_{1} \sqrt{(1-P) u_{i}^{n}} v_{i}^{n}+e a_{2} P u_{i}^{n} v_{i}^{n} .
\end{gathered}
$$



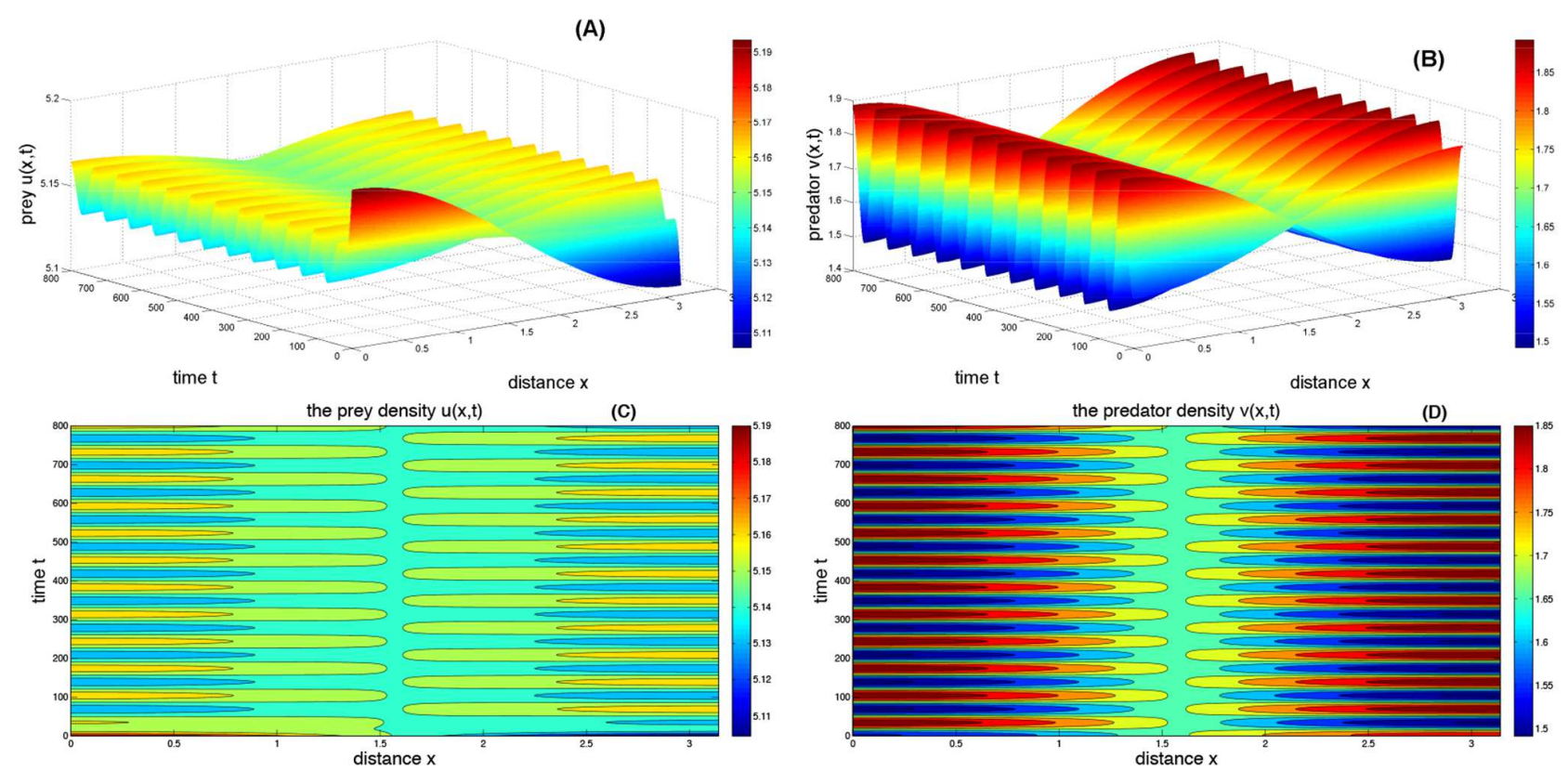

Figure 10. Numerical simulation of the system (1.4) when the equilibrium state $\left(u^{*}, v^{*}\right)=$ $(5.1494,1.6912)$ is unstable and the existence of a non homogeneous periodic solution for the values $k=44$ and $d=0.02 ; \widetilde{d}=0.01 ; a_{1}=0.5 ; a_{2}=0.7 ; P=0.5 ; l=1 e=0.5 ; m=0.5 ; r=$ 1.2 with the initial data $u(0, x)=u^{*}-0.015+0.06 \cos x$ and $v(0, x)=v^{*}-0.2+0.06 \cos 0.04 x$.
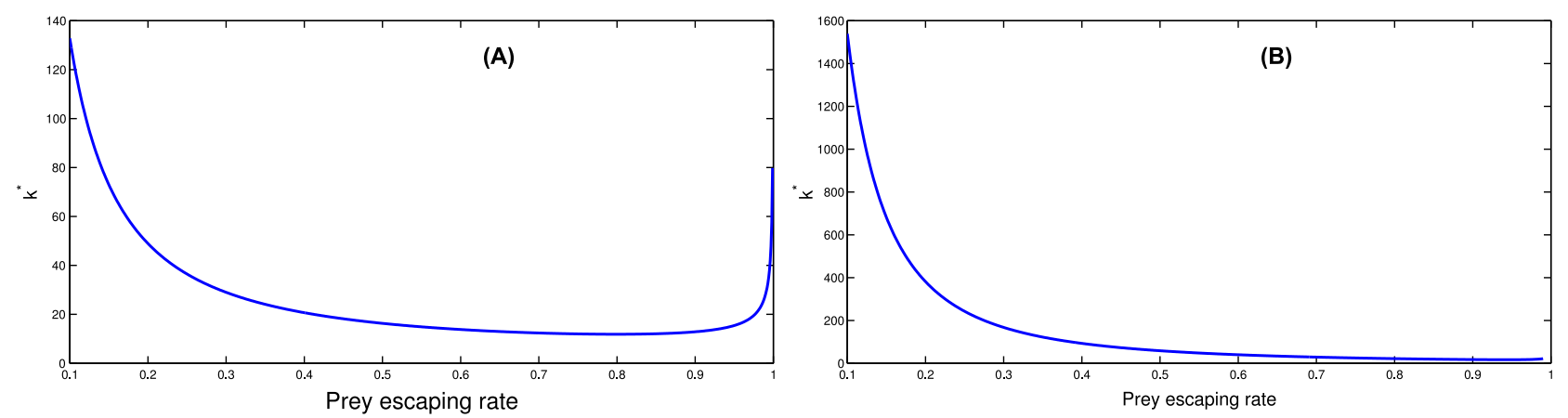

FIGURE 11. Numerical simulation of the effect of the prey escaping rate $P$ on the Hopf bifurcation value shown in (3.6), the values $e=0.44 ; m=1.01 ; r=0.2 ; k=8000$ are used, where for (A) we take $a_{1}=1 ; a_{2}=2.1 ;$ and for $(\mathrm{B})$ we take $a_{1}=2.5$ and $a_{2}=2.1$.

Figure 8: Represents the local stability of the interior equilibrium state $\left(u^{*}, v^{*}\right)$ for $k=32<k(0)=33.4344$ this result has been obtained in theorem 4.3, where (C) and (D) represents the projection of the surfaces (A) and $(\mathrm{B})$ on the plan $t-x$, respectively.

Figure 9: Represents the existence of a homogeneous periodic orbits for $k=34>k(0)=33.4344$ (means that $n=0)$ shown in theorem 4.3, where (C) and (D) represents the projection of the surfaces (A) and (B) on the plan $t-x$, respectively.

Figure 10: Represents the existence of a non homogeneous periodic orbits for $k>k(1)=43.3963$ (means that $n=1$ ) shown in theorem 4.3, where (C) and (D) represents the projection of the surfaces (A) and (B) on the plan $t-x$, respectively. 
Now shall we discuss the direction of Hopf bifurcation using a numerical simulation. Taking the following values $d=0.02 ; \widetilde{d}=0.01 ; a_{1}=0.5 ; a_{2}=0.7 ; P=0.5 ; l=1 ; e=0.5 ; m=0.5 ; r=1.5$. Thus we have:

(i) The set of all bifurcation points is $\Lambda=\{k(0), k(1), k(2)\}$ where $k(0)=22.1027, k(1)=25.4397, k(2)=$ 46.5027 .

(ii) For $k=k(1)$ we have $a_{11}=0.0324, a_{21}=0.1280, a_{12}=-1$ and $\omega=0.3576, a_{1}=1, b_{1}=-0.0012+$ $2.7939 i, a_{1}^{*}=-0.1677-0.0051 i, b_{1}^{*}=0.1677+0.0051 i$. Thus by a straight forward calculation, and by replacing those results in (4.30) (see also (4.31), (4.32), (4.33)) we obtained that $\operatorname{Re}\left\{c_{1}(0)\right\} \approx-0.8205<0$. Then the the periodic solutions are stables at $k=k(1)$.

\section{Discussion}

In this paper, a study of a new approach of escaping prey from a pack with a predator-prey interaction in the presence of spatial diffusion and subject to the homogeneous Neumann boundary conditions. The impact of the prey escaping on the positive equilibrium state $\left(u^{*}, v^{*}\right)$ has been successfully studied in the second section, where the negative effect on the prey density equilibrium has been obtained (see Fig. 1). This results shows that this specific behavior of the prey population has always a negative impact on the evolution of the prey density. On the other hand, the effect of the variable $P$ on the predator density equilibrium has been divided between a positive and negative effect, where it depends on two parameters the carrying capacity of the space of the prey $k$ and a critical value of the prey escaping rate denoted by $P_{c r}$. Also those results were justified using numerical simulations (Fig. 2). Because of the dependence of the predator density equilibrium (see Lem. 2.1) on two parameters $\mathrm{P}$ and $\mathrm{k}$ we presented in Figure 3 which shows the effect of those two parameters on the density equilibrium of the predator. Besides, in Figure 11 the impact of the variable $P$ on the value of the Hopf bifurcation shown in (3.6) which prove the huge sensitivity of the Hopf bifurcation value to the escaping prey rate. Mathematically, this sensitivity can lead to a huge variation in the solution behavioral of the considered systems (1.3) and (1.4).

In Section 3, our main focus has been on the study of the non spatial system (1.3). Firstly, the existence of the upper bound of solution has been successfully established. This upper bound has been used to prove the global stability of the semi trivial equilibrium state $(k, 0)$ in the absence of the interior equilibrium state $\left(u^{*}, v^{*}\right)$ (which means if $k<u^{*}$ ) the result has been also justified by a numerical simulation (Fig. 4). Besides, the local stability of the positive equilibrium state $\left(u^{*}, v^{*}\right)$ has been also determined, where a critical value of the carrying capacity of the space for the prey denoted by $k^{*}$ such that for $k<k^{*}$ the interior equilibrium is locally stable and unstable for $k>k^{*}$. Further, we obtained that the system (1.3) undergoes a Hopf bifurcation at $k=k^{*}$. This result has been checked out using numerical simulations (Figs. 5 and 6).

In the next section, the analysis of the diffusive system has been investigated. The characteristic equation has been calculated for the interior equilibrium state $\left(u^{*}, v^{*}\right)$. By analyzing the characteristic equation, it has been proved that the diffusive system undergoes a Hopf bifurcation at multi value $k=k(n)$ (where $n<N_{1}$ ) and the sequence $k(n)$ is increasing in $n$ (see Fig. 7). Note that for $n=0$ a homogeneous periodic solution appears (see Fig. 9) and non homogeneous periodic solution appears for $n=1,2, \ldots, j_{N^{*}}$ (see Fig. 10) (which appears when the interior equilibrium state $\left(u^{*}, v^{*}\right)$ is unstable).For determining the stability of the periodic solution the normal form on the center manifold theory has been used (see [4]). An important quantity denoted by $v_{2}$ shows the stability of the homogeneous and non homogeneous periodic solutions has been determined, where for $v_{2}<0$ the periodic solutions are unstable and stable for $v_{2}>0$.

Based on the biological meaning of those results, the main remark is the escaping rate never serves the prey population. In the real world, the objective of the attack of the predator on the prey pack is to divide this herd, where the main focus will be on hunting the escaping prey. This behavior can be seen widely in the real world, where the most hunted prey are the youngest ones. In other word, the prey must stay organized, and the pack is the easiest and effective way for prey to survive in the wild. Besides, the global stability of the semi trivial equilibrium $(k, 0)$ for $k<u^{*}$ means that for the smaller species the predator cannot satisfies it need (the prey is no more available to the predator), which leads to the extinction of the predator population. On the other hand, after the analysis of the system there exists a critical value of the carrying capacity of the space for the prey 
population such that for the small spaces (means that the carrying capacity is lower then this critical value) we can conserve the two populations at a constant density in the long term, and for large spaces (means that the carrying capacity is bigger then this critical value) we conserve the two population but with a periodic patterns.

\section{REFERENCES}

[1] V. Ajraldi, M. Pittavino and E. Venturino, Modeling herd behavior in population systems. Nonlin. Anal. Real World Appl. 12 (2011) 2319-2338.

[2] A. Bahar and X. Mao, Stochastic delay Lotka-Volterra model. J. Math. Anal. Appl. 292 (2004) 364-380.

[3] P.A. Braza, Predator-prey dynamics with square root functional responses. Nonlin. Anal. Real World Appl. 13 (2012) 18371843.

[4] J. Carr, Applications of Center Manifold Theory. SpringerVerlag, New York (1981).

[5] S.N. Chow and J.K. Hale, Methods of Bifurcation Theory. Springer, New York (1982).

[6] E. Cagliero and E. Venturino, Ecoepidemics with infected prey in herd defense: the harmless and toxic cases. Int. J. Comput. Math. 93 (2016) 108-127.

[7] S. Djilali, Herd behavior in a predator-prey model with spatial diffusion: bifurcation analysis and Turing instability. J. Appl. Math. Comp. 58 (2017) 125-149.

[8] S. Djilali, Impact of prey herd shape on the predator-prey interaction. Chaos Solitons Fractals 120 (2019) $139-148$.

[9] S. Djilali, Effect of herd shape in a diffusive predator-prey model with time delay. J. Appl. Anal. Comput. 9 (2019) 638-654.

[10] S. Djilali and S. Bentout, Spatiotemporal patterns in a diffusive predator-prey model with prey social behavior. Acta Appl. Math. (2019) DOI:10.1007/s10440-019-00291-z.

[11] S. Djilali, T.M. Touaoula and S.E.H. Miri, A Heroin epidemic model: very general non linear incidence, treat-age, and global stability. Acta Appl. Math. 152 (2017) 171-194.

[12] B. Hassard, N. Kazarinoff and Y. Wan, Theory and Applications of Hopf Bifurcation. Cambridge University Press, Cambridge (1981).

[13] Y. Huang, F. Chen and L. Zhong, Stability analysis of a prey-predator model with Holling type III response function incorporating a prey refuge. Appl. Math. Comput. 182 (2006) 672-683.

[14] G. Huang, W. Ma and Y. Takeuchi, Global properties for virus dynamics model with Beddington-DeAngelis functional response. Appl. Math. Lett. 22 (2009) 1690-1693.

[15] W. Ko and K. Ryu, Qualitative analysis of a predator-prey model with Holling type II functional response incorporating a prey refuge. J. Differ. Equ. 231 (2006) 534-550.

[16] Y. Kuang and E. Beretta, Global qualitative analysis of a ratio-dependent predator-prey system. J. Math. Biol. 36 (1998) 389-406.

[17] C. Li, A diffusive Holling-Tanner prey-predator model with free boundary. Int. J. Biomath. 11 (2018) 1850066.

[18] Z. Ma and S. Wang, A delay-induced predator-prey model with Holling type interaction functional response and habitat complexity. Nonlin. Dyn. 93 (2018) 1519-1544.

[19] I. Martina and E. Venturino, Shape effects on herd behavior in ecological interacting population models. Math. Comput. Simul. 141 (2017) 40-55.

[20] Q. Meng and L. Yang, Steady state in a cross-diffusion predator-prey model with the Beddington-DeAngelis functional response. Nonlinear Anal. Real World Appl. 45 (2019) 401-413.

[21] A. Mousaoui, S. Bassaid and E.H.A. Dads, The impact of water level fluctuations on a delayed prey-predator model. Nonlin. Anal. Real World Appl. 21 (2015) 170-184.

[22] K. Ryu, W. Ko and M. Haque, Bifurcation analysis in a predator-prey system with a functional response increasing in both predator and prey densities. Nonlin. Dyn. 94 (2018) 1639-1656.

[23] H.-B. Shi, W.-T. Li and G. Lin, Positive steady states of a diffusive predator-prey system with modified Holling-Tanner functional response. Nonlin. Anal. Real World Appl. 11 (2010) 3711-3721.

[24] Y. Song and X. Tang, Stability, steady-state bifurcation, and Turing patterns in predator-prey model with herd behavior and prey-taxis. Stud. Appl. Math. 139 (2017) 371-404.

[25] Y. Song and X. Zou, Spatiotemporal dynamics in a diffusive ratio-dependent predator-prey model near a Hopf-Turing bifurcation point. Comput. Math. Appl. 67 (2014) 1978-1997.

[26] X. Tang and Y. Song, Bifurcation analysis and Turing instability in a diffusive predator prey model with herd behavior and hyperbolic mortality. Chaos Solit. Frac. 81 (2015) 303-314.

[27] X. Tang and Y. Song, Turing-hopf bifurcation analysis of a predator-prey model with herd behavior and cross-diffusion. Nonlinear Dyn. 86 (2016) 73-89.

[28] E. Venturino, A minimal model for ecoepidemics with group defense. J. Biol. Syst. 19 (2011) 763-785.

[29] E. Venturino, Modeling herd behavior in population systems. Nonlin. Anal. Real World Appl. 12 (2013) $2319-2338$.

[30] E. Venturino and S. Petrovskii, Spatiotemporal behavior of a prey-predator system with a group defense for prey. Ecol. Complex. 14 (2013) 37-47.

[31] S. Wiggins, Introduction to Applied Nonlinear Dynamical Systems and Chaos. Springer-Verlag, New York (1991).

[32] Z. Xu and Y. Song, Bifurcation analysis of a diffusive predator-prey system with a herd behavior and quadratic mortality. Math. Meth. Appl. Sci. 38 (2015) 2994-3006. 
[33] C. Xu, S. Yuan and T. Zhang, Global dynamics of a predator-prey model with defense mechanism for prey. Appl. Math. Lett. 62 (2016) 42-48.

[34] R. Yang, M. Liu and C. Zhang, A diffusive predator-prey system with additional food and intra-specific competition among predators. Int. J. Biomath. 11 (2018) 1850060.

[35] W. Yang, Analysis on existence of bifurcation solutions for a predator-prey model with herd behavior. Appl. Math. Model. 53 (2018) 433-446.

[36] S. Yuan, C. Xu and T. Zhang, Spatial dynamics in a predator-prey model with herd behavior. Chaos 23 (2013) 033102.

[37] J. Zhang, W. Li and X. Yan, Hopf bifurcation and Turing instability in spatial homogeneous and inhomogeneous predator-prey models. Appl. Math. Model. 218 (2011) 1883-1893.

[38] H. Zhu and X. Zhang, Dynamics and patterns of a diffusive prey-predator system with a group defence for prey. Disc. Dyn. Nat. Soc. 2018 (2018) 6519696. 